\title{
A Critical Assessment of Methods for the
}

\section{Intrinsic Analysis of Liquid Interfaces: 1.}

\section{Surface Site Distributions}

\author{
Miguel Jorge ${ }^{* \dagger}$, Pál Jedlovszky $y^{\ddagger}$ and M. Natália D. S. Cordeiro ${ }^{\S}$ \\ ${ }^{\dagger}$ LSRE/LCM - Laboratory of Separation and Reaction Engineering, Faculdade de \\ Engenharia da Universidade do Porto, Rua Dr. Roberto Frias, 4200-465 Porto, Portugal \\ ${ }^{\ddagger}$ Laboratory of Interfaces and Nanosize Systems, Institute of Chemistry, Eotvos Loránd \\ University, Pázmány Péter stny. 1/a, H-1117 Budapest, Hungary \\ and
}

HAS Research Group of Technical Analytical Chemistry, Gellért tér 4, H-1111

Budapest, Hungary

and

EKF Department of Chemistry, H-3300 Eger, Leanyka u. 6, Hungary

${ }^{\S}$ REQUIMTE, Faculdade de Ciências da Universidade do Porto, Rua do Campo Alegre, 687, 4169-007 Porto, Portugal

Email address: mjorge@fe.up.pt

Title Running Head: Intrinsic Analysis of Liquid Interfaces 


\begin{abstract}
Substantial progress in our understanding of interfacial structure and dynamics has stemmed from the recent development of algorithms that allow for an intrinsic analysis of fluid interfaces. These work by identifying the instantaneous location of the interface, at the atomic level, for each molecular configuration and then computing properties relative to this location. Such a procedure eliminates the broadening of the interface caused by capillary waves and reveals the underlying features of the system. However, a precise definition of which molecules actually belong to the interfacial layer is difficult to achieve in practice. Furthermore, it is not known if the different intrinsic analysis methods are consistent with each other and yield similar results for the interfacial properties. In this paper, we carry out a systematic and detailed comparison of the available methods for intrinsic analysis of fluid interfaces, based on a molecular dynamics simulation of the interface between liquid water and carbon tetrachloride. We critically assess the advantages and shortcomings of each method, based on reliability, robustness and speed of computation, and establish consistent criteria for determining which molecules belong to the surface layer. We believe this will significantly contribute to make intrinsic analysis methods widely and routinely applicable to interfacial systems.
\end{abstract}

$\underline{\text { Key words: }}$ Water/organic interfaces, Intrinsic surface, Statistical Mechanics, Molecular Simulation.

\title{
1. Introduction
}

Interfaces involving fluid phases (be it between a liquid and a gas or between two immiscible liquids) have fascinated scientists for centuries [1]. They are ubiquitous in nature and assume a critical role in a wide variety of chemical, physical, biological and environmental processes [2]. In recent years, our knowledge of the molecular-level structure and properties of liquid interfaces has progressed dramatically. This is due to remarkable developments in experimental techniques, like non-linear spectroscopic methods [1] or X-ray scattering [3], but 
also due to advances in theoretical methods, particularly molecular simulation [2]. Despite such advances, the analysis of liquid interfaces is still complicated by the fact that they are inherently rough, i.e., the surface of a given fluid is corrugated by thermal fluctuations, or capillary waves [4]. In fact, the observed (or "global") average profile of a given property (say, density) is normally sampled on the basis of the average cross-section of the system,

$$
\rho_{G}(z)=\left\langle\frac{1}{A_{0}} \sum_{i=1}^{N} \delta\left(z-z_{i}\right)\right\rangle,
$$

where $N$ is the number of molecules, $z_{i}$ is their coordinate along the axis perpendicular to the interface, and $A_{0}$ is the nominal cross-sectional area. Due to the effect of capillary waves, the global profile will be smoothed by the instantaneous fluctuations of the position of the interface itself. In fact, one can consider the existence of an intrinsic profile upon which the effect of thermal fluctuations is felt, leading to the observed global profile - this is, in fact, one of the cornerstones of capillary wave theory (CWT) [5]. Such an intrinsic profile is given by

$$
\rho_{I}(z)=\left\langle\frac{1}{A_{0}} \sum_{i=1}^{N} \delta\left(z-z_{i}+\xi\left(x_{i}, y_{i}\right)\right)\right\rangle,
$$

where $\xi$ is the instantaneous position of the surface, and $x_{i}$ and $y_{i}$ are the molecular coordinates in the plane parallel to the interface.

In the majority of theoretical treatments of liquid interfaces, the intrinsic profile was assumed to take a specific functional form, which may range from a simple step function, as in the original theory [5], to a density functional description of the fluid [6]. An interesting alternative, however, is to extract the intrinsic density profile directly from simulations of interfacial systems. This would naturally require a protocol for identifying the instantaneous position of the surface in each molecular configuration, relative to which the profile would be averaged, following (2). The first attempt at such a protocol for a liquid-vapor system is due to 
Stillinger [7], and is based on defining interfacial molecules as those that are in direct contact with a percolating volume of empty space representing the vapor phase. Such a definition, however, turns out to be extremely difficult to implement in practice, and it took more than twenty years until the first computationally tractable definition of the surface layer appeared, in the form of the Intrinsic Sampling Method (ISM) of Chacón and Tarazona [8]. Other methods have later appeared [9-12] allowing one to obtain unprecedented detailed information about the intrinsic structure, density profiles, hydrogen bond network, molecular orientations, diffusivity, as well as other properties of fluid interfaces.

One aspect of intrinsic analyses that generates a wide consensus is that it is difficult to conclusively establish which molecules actually belong to the surface layer, which is mainly due to the inherently fluid nature of the phases under consideration. It should be noted that a recent method proposes a way to obtain the location of the intrinsic surface without requiring the identification of a set of interfacial molecules [12]. Nevertheless, most intrinsic analysis methods [8-11] rely on identifying the interfacial molecules and then constructing a mathematical surface based on their positions, to enable the computation of intrinsic profiles from (2). Naturally, there are many alternative ways to achieve this. In this context, it is of the utmost importance to establish if each method indeed provides an intrinsic view of the interface, and if the results obtained using different methods are consistent with each other - this is the task we propose to accomplish. In this paper, we present results of a comparison of four different methods for identifying the set of surface molecules from simulation data. We have chosen as a prototype system the liquid/liquid interface between water and carbon tetrachloride (simulation details are given in section 2), because it is a realistic system but simple enough to ensure computational tractability. Each method is first studied individually in detail (sections 3.1 to 3.4 ) in terms of their validity, advantages and disadvantages, leading, in several cases, to improvements in their respective protocols. We then critically compare all methods, based on reliability, robustness and speed of computation, in section 3.5 of the paper. Section 4 presents the main conclusions of our study. 


\section{Simulation Details}

Molecular dynamics simulation of the water/ $\mathrm{CCl}_{4}$ liquid-liquid interface has been performed in the canonical $(N, V, T)$ ensemble at the temperature of $298 \mathrm{~K}$. The system consisted of 4000 water and $2000 \mathrm{CCl}_{4}$ molecules. The $x, y$ and $z$ edges of the basic box were 5.0, 5.0 and $17.9 \mathrm{~nm}$ long, respectively, $z$ being the surface normal axis. Standard periodic boundary conditions have been applied. The water and $\mathrm{CCl}_{4}$ molecules were described by the rigid, four site TIP4P model [13] and a rigid five-site model of McDonald et al., [14] respectively. Thus, the total potential energy of the system has been calculated as the sum of the interaction energies of the molecule pairs, the latter being the sum of a Lennard-Jones and a charge-charge Coulomb term. All interactions have been truncated to zero beyond the molecule center-based cut-off distance of $14.0 \AA$ A.

The simulation was started from a configuration generated in a previous study. [15] The simulation was performed with the GROMACS program package. [16] The geometry of the individual $\mathrm{CCl}_{4}$ and water molecules was kept unchanged using the SHAKE [17] and SETTLE [18] algorithms, respectively. The temperature of the system was controlled by means of the Berendsen thermostat. [19] The long range-part of the Coulomb interactions was accounted for using the Particle Mesh Ewald (PME) method. [20] The equations of motion were integrated in time steps of $2 \mathrm{fs}$, and the system was equilibrated for $1 \mathrm{~ns}$. Then 2000 sample configurations, separated from each other by 0.5 ps long trajectories each, were saved for the analyses. These sampled configurations were translated along the interface normal axis $z$ in such a way that the position of the center-of-mass of the water molecules was moved to the origin of the coordinate axes. All results were averaged over the 2000 sampled configurations.

\section{Results and Discussion}

\subsection{The Intrinsic Sampling Method of Chacón and Tarazona}

The Intrinsic Sampling Method (ISM) was originally developed by Chacón and Tarazona $[8,21,22]$ for the analysis of liquid/vapor interfaces of simple model fluids (such as the Lennard-Jones or the soft-alkali models). It was subsequently applied to the water surface [23] 
and recently adapted for the characterization of liquid/liquid interfaces [24,25]. The underlying idea is to define the intrinsic interface as the minimal area surface that goes through a set of pivot sites, which are essentially the atomic sites located at the interface. If the coordinates of those surface sites are defined by $\left(\mathbf{R}_{i}, z_{i}\right)=\left(x_{i}, y_{i}, z_{i}\right)$, then a smooth mathematical function passing through all the coordinates of the pivot sites can be constructed in terms of a sum of Fourier components, as follows:

$$
\xi\left(\mathbf{R}, q_{m}\right)=\sum_{0 \leq\left\{\mathbf{q} \mid \leq q_{m}\right.} \hat{\xi}_{q} e^{i \mathbf{q} \mathbf{R}}=\sum_{\mu^{2}+v^{2} \leq n_{M}^{2}} a_{\mu v} f_{\mu}(x) f_{v}(y),
$$

where $\mathbf{q}$ is the wavevector, with maximum wavevector cutoff $q_{m}$. The second expression is a more useful representation in terms of sines/cosines, with $f_{0}(x)=0, f_{\mu}(x)=\cos \left(2 \pi \mu x / L_{x}\right)$, and $f_{-\mu}(x)=\sin \left(2 \pi \mu x / L_{x}\right)$ for $\mu>0$, where $a_{\mu \nu}$ are real coefficients with indices running from $-n_{M}$ to $n_{M}$. In Chacón and Tarazona's papers [8,21-25], $n_{M}$ is always set to $\approx L_{x} / \sigma$, where $\sigma$ is a characteristic atomic diameter, such that all possible wavevectors down to atomic resolution are used.

Subject to the minimal area requirement, the intrinsic surface is obtained by minimizing the function [24]

$$
W=\frac{1}{2} \sum_{i=1}^{N_{S}}\left(z_{i}-\xi\left(\mathbf{R}_{i}\right)\right)^{2}+\frac{\phi L_{x}^{2}}{2} \sum_{|\mathbf{q}| \leq q_{m}} q^{2}\left|\hat{\xi}_{q}\right|^{2}
$$

where $N_{S}$ is the total number of surface sites, and $\phi$ is a parameter that sets a maximum threshold distance between the surface and the coordinates of the pivot sites. The optimal value of $\phi=10^{-8}$ suggested by the authors [24] represents a compromise between good numerical precision and physical realism. Combining equations (3) and (4), we obtain a set of linear equations with respect to the coefficients $a_{\mu v}$ : 
In this paper, equation (5) was solved using an efficient LU decomposition algorithm [26]. Once the coefficients are computed, the intrinsic surface follows directly from equation (3).

Using the above definition of the intrinsic surface, the ISM method focuses on finding the set of surface pivot sites in a self-consistent way. To obtain a first estimate of the pivot sites, the $(x, y)$ plane is divided into a grid of $N_{0} \times N_{0}$ squares, thus building a mesh of rectangular prisms with transverse size $L_{x} / N_{0}$. The atomic sites with the most external positions in each of these prisms, i.e., the sites that are closest to the opposite phase in a liquid/liquid system, are chosen as the initial pivot sites. Equation (5) is then solved to obtain the minimal area surface that passes through these pivot sites, and an iterative procedure follows to increase the number of interfacial atoms. In the original method [8,21], this was achieved by successively adding as pivots all sites that were closer to the surface than a predefined threshold distance. In a later paper [22], Chacón and Tarazona proposed an alternative criterion for deciding whether or not to incorporate new sites in the set of pivots. The authors observed that some configurations (particularly at higher temperatures and for anisotropic fluids like water) required values of the threshold distance that were unreasonably small, and suggested that using the surface layer density (defined in a dimensionless form by $n_{S}=N_{S} \sigma^{2} / L_{x}^{2}$ ) as a control parameter produced more robust results. Thus, the closest site to the surface is added in each iteration until the surface density reaches a predefined value. In the present paper, we employ this modified version of the ISM method.

We have applied the ISM method to analyze the water/CCl 4 interface. This analysis was carried out on a molecular basis, i.e., each molecule was considered as a whole, with water centered at the oxygen atom and $\mathrm{CCl}_{4}$ centered at the carbon atom. As characteristic site diameters, we take the Lennard-Jones diameters of the water oxygen and of the $\mathrm{CCl}_{4}$ carbon. 
Figure 1 shows the resulting number density distributions of interfacial molecules for several values of the control parameter, $n_{S}$. It can be seen that the increase in the density of surface molecules as $n_{S}$ increases is mostly felt on the inner side of the interface for both components the first interfacial molecules are those that are closest to the opposite phase, and then the interfacial layer grows by incorporating molecules that are progressively farther away from the opposite phase. For all studied values of $n_{S}$, the distributions of the positions of water molecules at the surface show an approximately Gaussian shape. However, the same cannot be said of the $\mathrm{CCl}_{4}$ distributions. They deviate from a Gaussian shape at low density, when insufficient molecules have been incorporated, and at high density, when molecules that belong to the second layer are counted as interfacial. The latter effect is clearly seen in the pronounced shoulder in the $\mathrm{CCl}_{4}$ distribution for $n_{S}=1.185$. In fact, the same effect is observed for water, although it can only be visually discerned for higher densities than those shown in Figure 1.

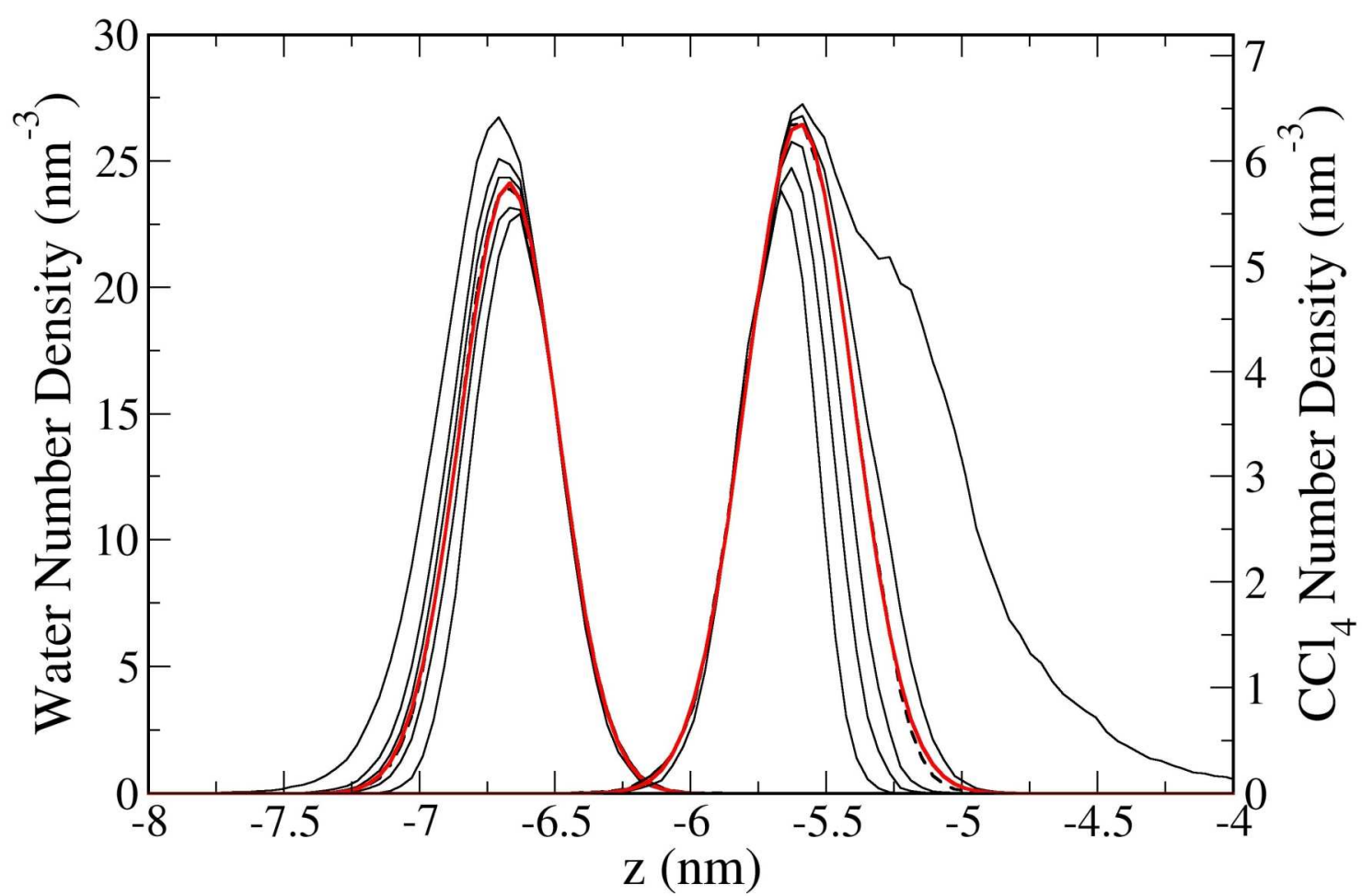

Figure 1 - Number density distributions of interfacial molecules for water (left curves and left axis) and $\mathrm{CCl}_{4}$ (right curves and right axis) obtained from the ISM with increasing values of the dimensionless surface layer density, from bottom to top: water $-0.895,1.018,1.082,1.130$, 
$1.193,1.392 ; \mathrm{CCl}_{4}-0.423,0.508,0.576,0.660,0.719,1.185$. The thick red lines are fits to a Gaussian distribution for selected curves (water $-1.082 ; \mathrm{CCl}_{4}-0.660$ ), which are shown as dashed lines. The curves for $\mathrm{CCl}_{4}$ are shifted by $0.6 \mathrm{~nm}$ to the right for clarity.

In order to more quantitatively assess the above effects, we have fitted the interfacial density distributions to a Gaussian function. Two of those fits are depicted as thick red lines in Figure 1, and the values of the correlation coefficients are plotted in Figure 2 as a function of $n_{S}$. For the organic component, the effect described above is very pronounced, and there is only a narrow region of $n_{S}$ values for which the distribution is truly Gaussian (between about 0.6 and 0.7 ), corresponding to a maximum in the correlation coefficient (at $n_{S}=0.66$ ). For water, the maximum (located at $n_{S}=1.13$ ) is much shallower, presumably because water is a smaller molecule for which the distinction between different molecular layers is more difficult to establish. Nevertheless, it is clear that there is an optimal range of surface densities, between about 1.0 and 1.2.

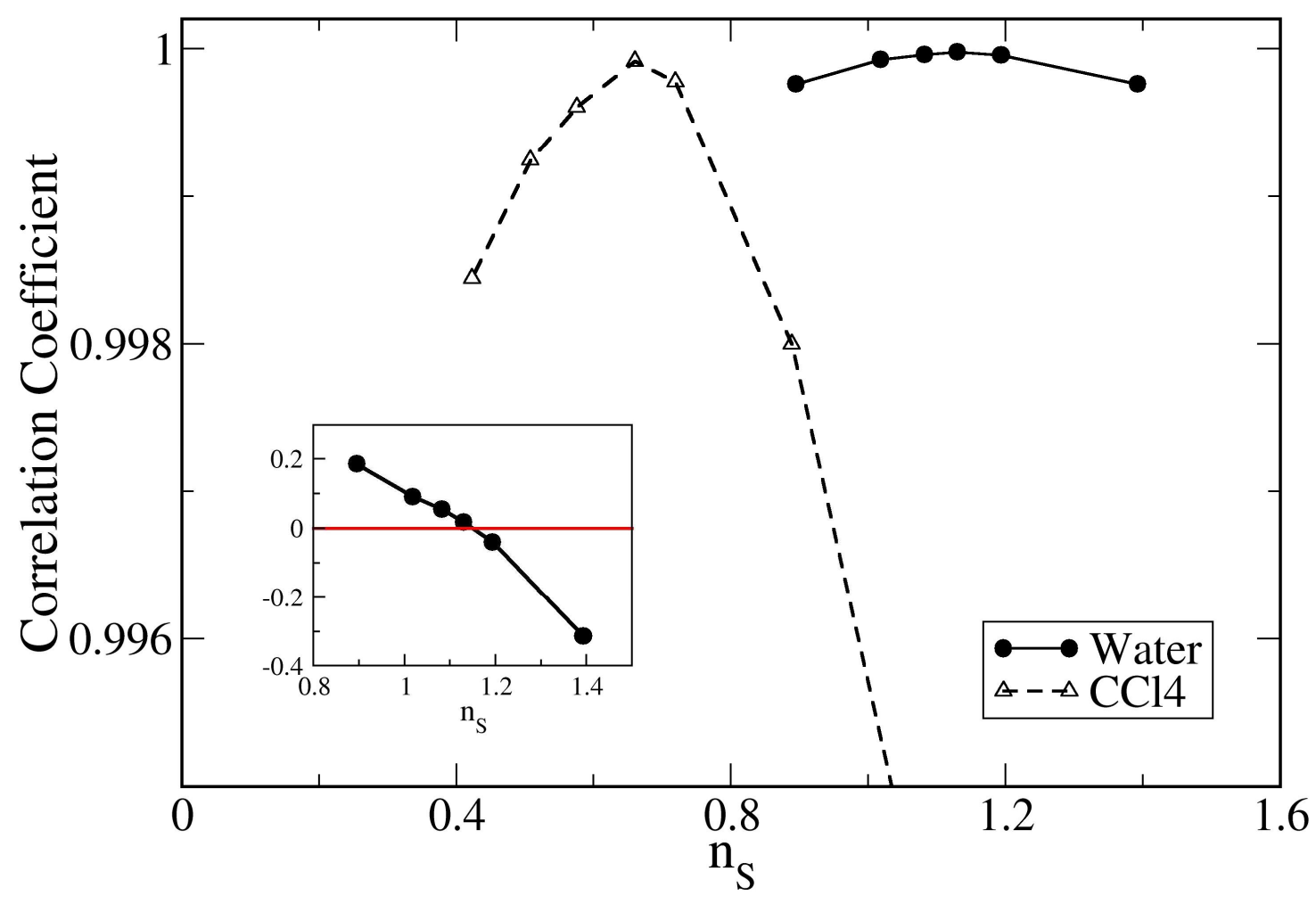


Figure 2 - Correlation coefficients obtained by fitting the interfacial density distributions obtained from the ISM to a Gaussian expression, as a function of the dimensionless surface layer density. The inset shows the skewness of the water distribution as a function of the dimensionless surface layer density.

The criterion for choosing the optimal surface density based on the best fit to a Gaussian distribution assumes that the density distribution of surface molecules obeys Gaussian statistics, which is supported by a recent theoretical study [27]. However, we consider the possibility that this may not always be the case. A more reasonable assumption could be to say that the interfacial distribution should be as symmetric as possible (but not necessarily Gaussian). With this idea in mind, we calculated the skewness of each distribution of water surface molecules as a function of the surface density, and the results are shown in the inset of Figure 2. As we can see, when the density is too low, the innermost surface molecules are excluded and the distribution is skewed to the right. On the other hand, when the density is too high, molecules from the second layer are added and the distribution becomes skewed to the left (see also Figure 1). The optimal point, with a skewness close to zero, is reached for $n_{S}=1.13$, which precisely matches the value obtained from the Gaussian fits.

It would be very useful to have another criterion for choosing the optimal value of $n_{S}$ that is not based on an analysis of the shape of the interfacial distributions. As we have mentioned previously, in the improved version of the ISM method [22] successive molecules are incorporated into the interfacial layer based on their proximity to the surface function, until $n_{S}$ reaches the predefined value. For the highest values of $n_{S}$ considered here, we have plotted the distance to the surface of each molecule that is iteratively added to the list $(\tau)$, averaged over all configurations of the trajectory. Such plots are shown in Figure 3 for both components, starting from the initial estimates of surface sites, i.e. the ones closest to the opposite phase. Each point of the curve corresponds to one iteration, and represents the distance between the new pivot site and the surface function calculated in the previous step of the method, as a function of the surface density $n_{S}$ at the instant of adding the new pivot site to the list. As expected, the distance to the surface increases monotonically as the surface density increases. 
However, some interesting insight can be obtained by analyzing the curvature of the plots. The first (convex) part of the curves shown in Figure 3 simply reflects the successive addition of molecules that are already very close to this first estimate of the surface. As $n_{S}$ increases further, $\tau$ increases gradually, but now with a negative curvature. At a certain stage, however, the curvature changes once more, until it levels off in a plateau for high values of $n_{S}$. This behavior reflects the layering structure of each phase. As successive molecules that truly belong to the interfacial layer are incorporated, $\tau$ becomes increasingly larger (concave shape) due to the addition of molecules that are at the inner edge of this layer. Then, molecules that belong to the outer edge of the second layer start to be added, and the plot becomes convex. The plateau corresponds to the addition of molecules that are near the center of the second layer, and whose addition already has little effect on the shape of the surface function.

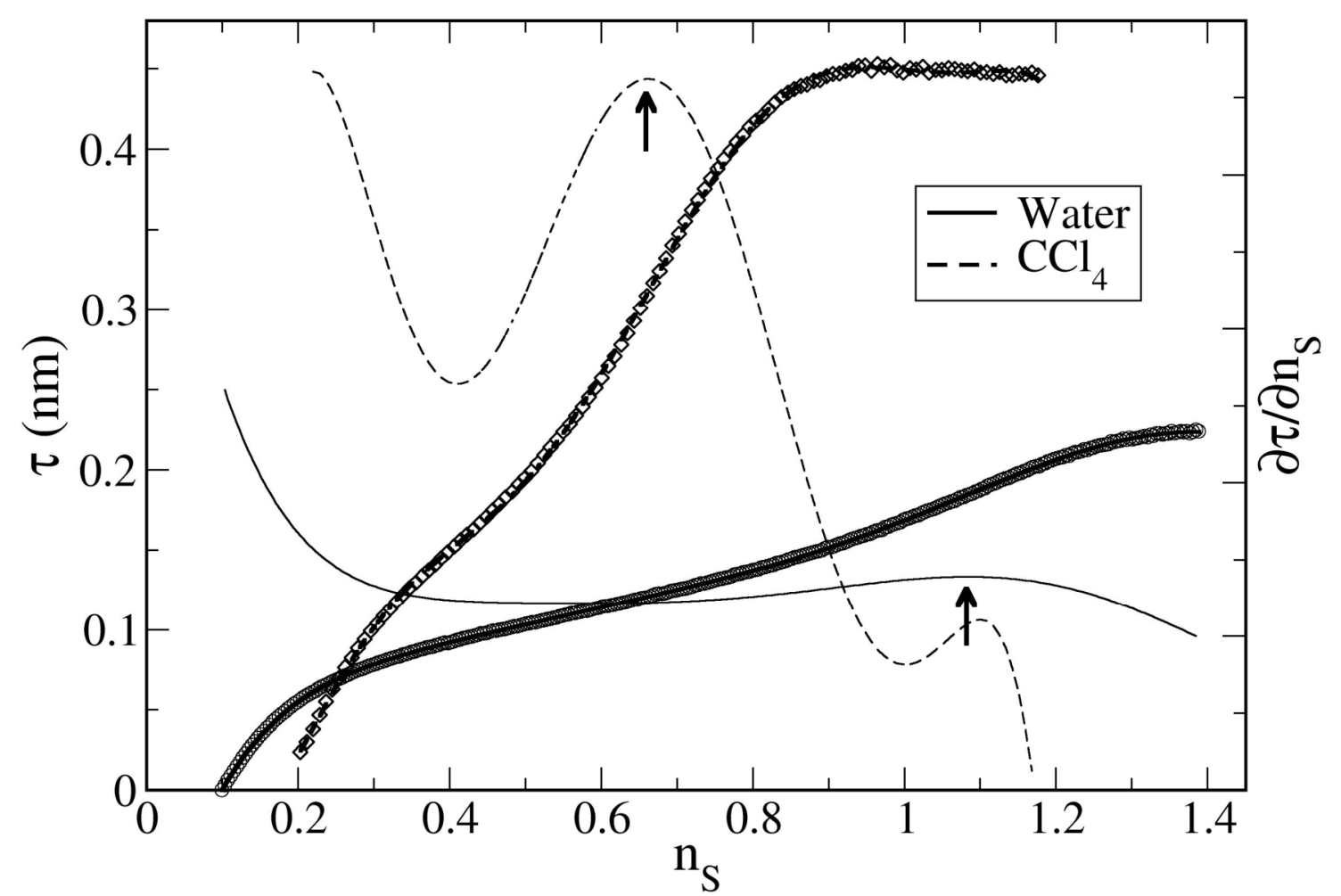

Figure 3 - Average distance between molecules successively incorporated into the interfacial layer during each ISM iteration and the surface function from the previous step, for water (circles and full lines) and $\mathrm{CCl}_{4}$ (diamonds and dashed lines). The thick lines (left axis) are fits 
of the data points (marked by symbols) to a polynomial of degree 7, and the thin lines (right axis) are obtained by differentiating the fitted polynomial.

Based on this analysis, it is reasonable to associate the change in slope with a separation between the interfacial layer and the second layer. To identify this point more precisely, we have fitted the data of Figure 3 to polynomial expressions (a polynomial of degree 7 was sufficient for an adequate description of the entire data set). The fits are shown as thick lines in Figure 3, while the derivatives of the fitted functions are shown as thin lines. Looking at the derivative, it is possible to clearly identify the inflection point for both water and $\mathrm{CCl}_{4}$ (the second maximum in the curve for $\mathrm{CCl}_{4}$ is merely an artifact of the statistical noise in the data at high surface density). The inflection points, marked with arrows in Figure 3, yield surface layer densities of 0.66 for $\mathrm{CCl}_{4}$ and 1.08 for water, which are both well within the optimal ranges found by analyzing the shape of the interfacial distributions (see Figure 2). As was observed in the analysis of the shape of the interfacial distributions, the inflection point is much sharper for the large organic molecules than for the small water molecules, for the reasons discussed above.

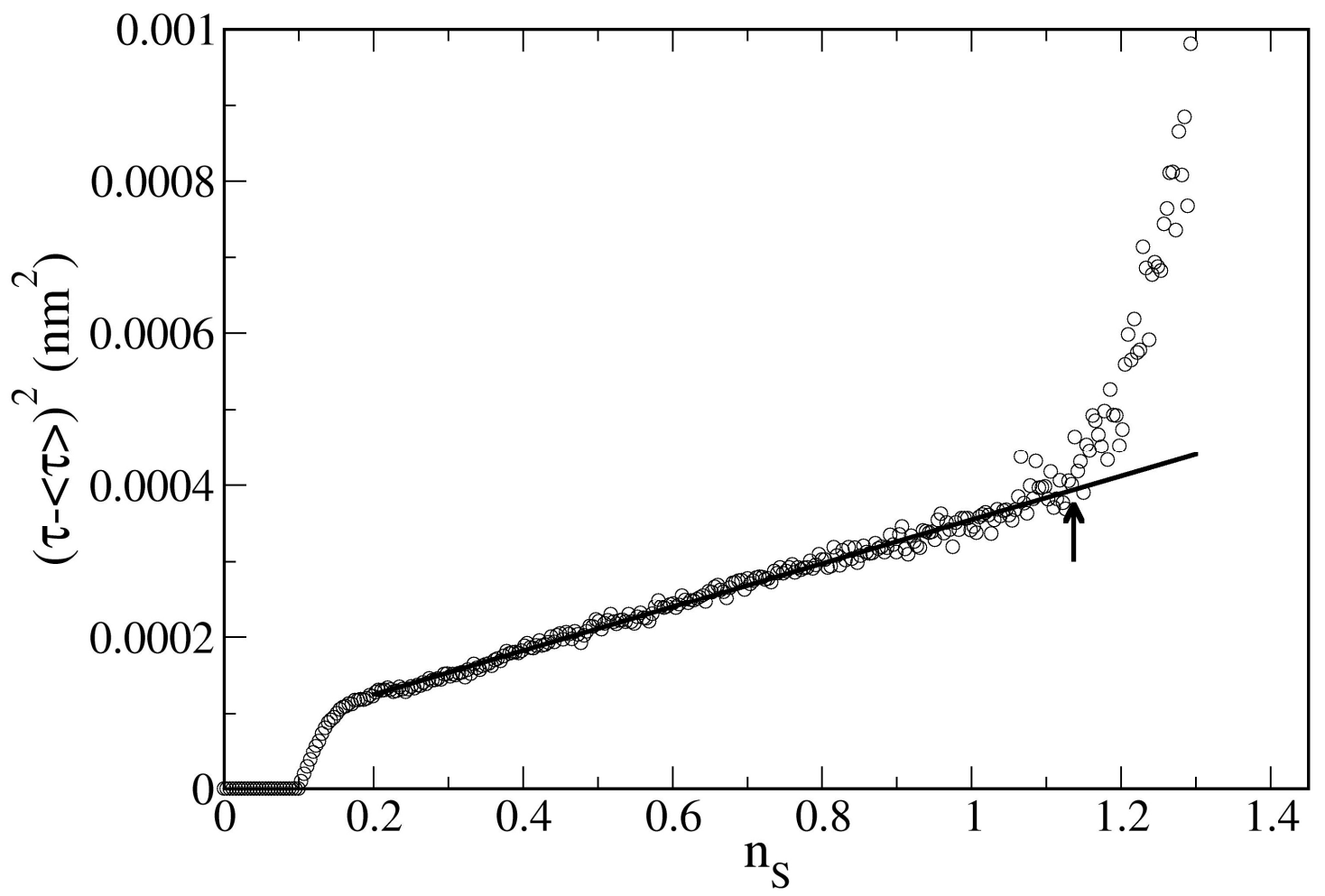


Figure 4 - Average fluctuations of the distance between molecules successively incorporated into the interfacial layer during each ISM iteration and the surface function from the previous step for water (circles). The thick line is a linear fit to the data points between 0.2 and 1.1. The arrow marks the point of departure from linear behavior.

The molecular layering at the interface manifests itself also in the fluctuations of the variable $\tau$. In Figure 4 we plot the quantity $\left\langle(\tau-\langle\tau\rangle)^{2}\right\rangle$ as a function of the surface layer density for the water surface (for the organic surface, the statistics are poor and it becomes difficult to discern a meaningful trend). After addition of the first few molecules, the fluctuations in $\tau$ increase linearly but then show a sharp increase at higher densities. This sharp increase arises from the fact that molecules from the second layer are being included as interfacial molecules, causing pronounced oscillations in the surface Fourier function and inducing strong fluctuations in $\tau$. The point at which the fluctuations show a strong increase is observed at approximately $n_{S}=1.14$, which agrees very closely with the optimal surface density obtained from the above analysis.

Our choice of optimal surface density based on the shape of the interfacial density distribution agrees very well with our analysis of $\tau$, shown in Figures 3 and 4 . Furthermore, our result for the water layer agrees very nicely with the values of 1.1 [23] and 1.15 [24] proposed by Tarazona and co-workers for the water/vapor interface on the basis of the shape of the intrinsic density profile, which further emphasizes the self-consistency of the ISM method. While this paper was being prepared, we became aware of a recent study by Chacón et al. [28] in which the authors propose alternative methods of determining the optimal surface layer density, based on the exchange rate of surface molecules or on the decay time of the autocorrelation function for the Fourier components of the intrinsic surface. The consistent results obtained by Chacón et al. using all the different methods are corroborated by our present analysis. 
Apart from $n_{S}$, there are a few parameters in the ISM that can be adjusted, and that may have an effect on the accuracy and efficiency of the method. We have performed a detailed analysis of $N_{0}$ and $n_{M}$, which is presented in Supporting Information, in order to optimize the performance of the method. It is possible to reduce the computational requirements by increasing the value of $N_{0}$ up to 7 without sacrificing the accuracy of the method (Figure S1). However, we adopt the conservative value of $N_{0}=5$ throughout the remainder of this paper. As for $n_{M}$, we have observed (Figure S2) that it cannot be reduced significantly below the guideline of $n_{M} \approx L_{x} / \sigma$ proposed by Tarazona and Chacón [21] - we have used the slightly lower values of 15 for water and 10 for $\mathrm{CCl}_{4}$. As pointed out by the authors themselves [21,28], a further reduction causes unphysical artifacts in the minimization procedure due to an overfitting effect, and consistent results are only obtained when the wavelength cutoff is fine-tuned at the site diameter.

\subsection{The Grid-based Intrinsic Profile method of Jorge and Cordeiro}

Since early molecular simulation studies of liquid/liquid interfaces $[29,30]$, the roughness of the interface, measured in terms of its average width and position, has been characterized using a grid-based method. Essentially, the plane parallel to the interface was divided into a grid of $N_{G} \times N_{G}$ squares. The local position and width of the interface were separately measured in each of the resulting rectangular prisms of transverse size $L_{x} / N_{G}$, and then averaged over all prisms. The variation of the shape of the position and width distributions with increasing grid resolution (set by the parameter $N_{G}$ ) was used to conclude that the interface is locally sharp but corrugated by thermal fluctuations, or capillary waves [30]. In fact, the idea of dividing the interfacial plane into a grid can be traced back to a theoretical paper by Weeks [31], which attempted to reconcile CWT with a density-functional theory description of the interface. In Weeks' paper [31], the resolution of the grid was only increased up to the value of the bulk correlation length, beyond which CWT loses physical meaning and is no longer applicable. 
The idea of using such a grid-based method to obtain density profiles that better reveal the underlying structure of liquid/liquid interfaces was pioneered by Fernandes et al. [32]. The resulting density profiles showed pronounced oscillations near the interface, but, because the restriction that the grid resolution should not go beyond the bulk correlation length was still imposed, it was not clear whether the method did indeed yield the true intrinsic profile. Recently, Jorge and Cordeiro $[9,33]$ extended this method and were able to obtain intrinsic density profiles that showed good agreement with profiles obtained independently by another group [11]. The key aspect consisted of increasing the grid resolution well beyond the bulk correlation length, down to atomic sizes. This is consistent with the analysis of Tarazona and co-workers $[8,22]$, who unequivocally established the need to go beyond the range of validity of CWT in order to obtain true intrinsic density profiles. The optimum grid resolution was found to be approximately given by $N_{G} \approx L_{x} / \sigma$, where $\sigma$ was the diameter of the largest atomic site in each liquid $[9,33]$. Hereafter, we will call this method the Grid-based Intrinsic Profile (GIP) method.

Although the GIP method was not specifically designed to identify the complete set of interfacial molecules, it is interesting to analyze to which extent it is able to do so. For this purpose, we identify the interfacial molecules based on the limits of each phase, determined using the grid-based method - in each prism, the atomic site closest to the opposite phase was found, and the molecule to which that site belongs was labeled as interfacial. The GIP method was applied in this way to the water $/ \mathrm{CCl}_{4}$ interface and the resulting number density distributions of interfacial molecules are shown in Figure 5 for several values of the control parameter $N_{G}$. As expected, the average surface density increases with $N_{G}$, since the method incorporates molecules that are further within each phase when the grid resolution increases. As observed above for the ISM, the distributions have an approximately Gaussian shape, except at very low resolution, when insufficient molecules are incorporated, and at high resolutions, where significant deviations are observed. For water, we can clearly see that the distributions develop a long tail extending further into the bulk phase as the resolution increases, while for 
$\mathrm{CCl}_{4}$ the effect is even more evident, in the form of a pronounced shoulder. As for the ISM, this effect is due to the incorrect consideration of molecules that actually belong to the second molecular layer as interfacial molecules.

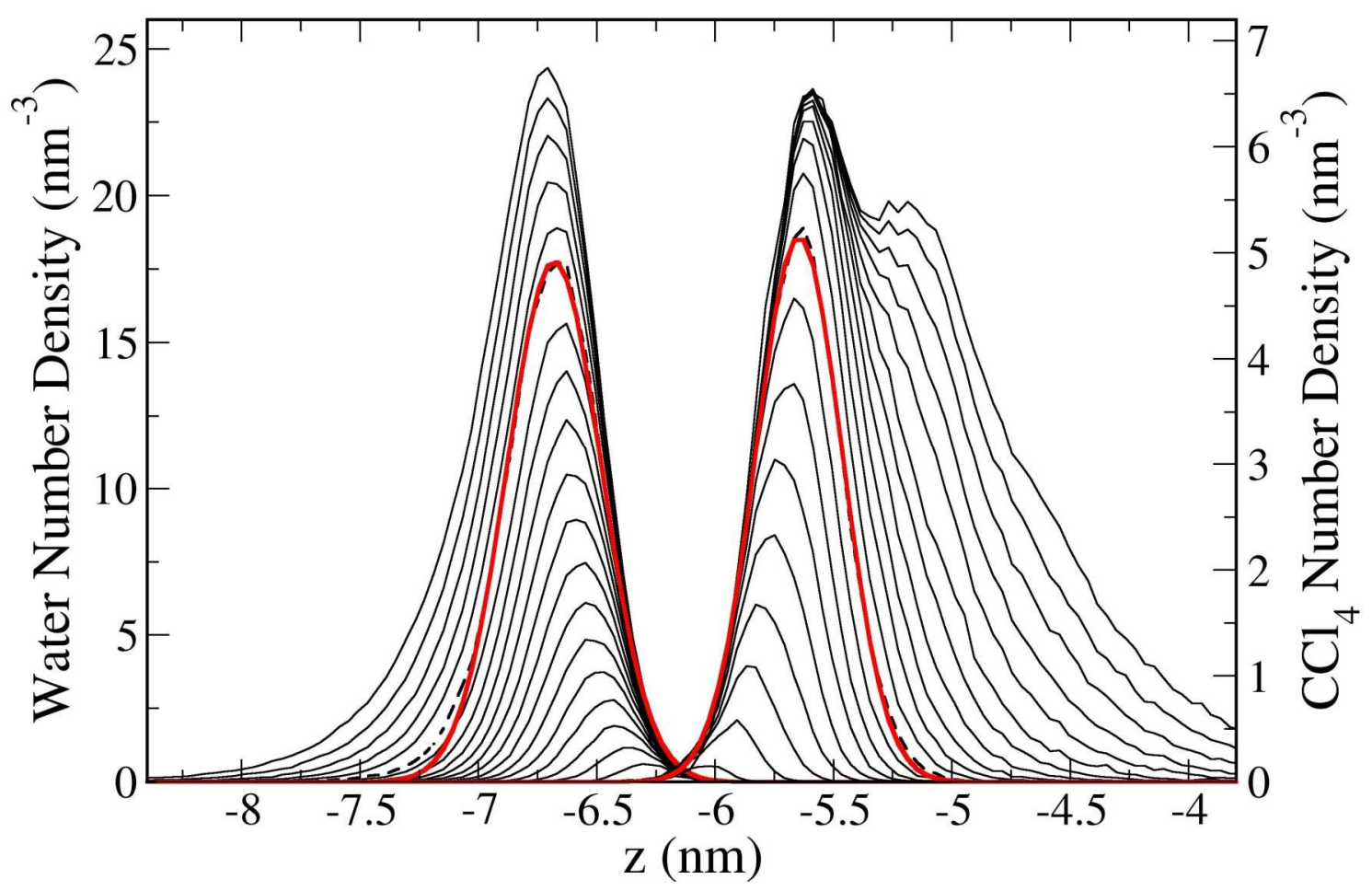

Figure 5 - Number density distributions of interfacial molecules for water (left curves and left axis) and $\mathrm{CCl}_{4}$ (right curves and right axis) obtained from the GIP method with increasing values of the grid resolution $-N_{G}$ increases from 1 to 20 in increments of 1 from bottom to top. The thick red lines are fits to a Gaussian distribution for selected curves (water $-15 ; \mathrm{CCl}_{4}-9$ ), which are shown as dashed lines. The curves for $\mathrm{CCl}_{4}$ are shifted by $0.6 \mathrm{~nm}$ to the right for clarity.

In analogy with the optimal surface density, $n_{S}$, in the ISM, we expect there to exist an optimal value of $N_{G}$ that gives the best possible representation of the interfacial layer. Based on a previous analysis of the density profiles for other interfaces, Jorge and Cordeiro [9,33] concluded that the optimal value of $N_{G}$ should be given approximately by $N_{G} \approx L_{x} / \sigma$. Using this rule for our system yields $N_{G} \approx 16$ for water and $N_{G} \approx 11$ for $\mathrm{CCl}_{4}$ (rounding to the nearest 
integer in both cases). By integrating the respective interfacial density distributions we obtain the following values for the dimensionless surface density: $n_{S}=1.02$ for water and $n_{S}=0.64$ for $\mathrm{CCl}_{4}$. The latter result is close to the value obtained using the ISM procedure $(0.66)$, but the result for water is somewhat lower than the ISM value (1.13).

Alternatively, one may consider that the optimal value of $N_{G}$ should afford the maximum possible resolution provided the distributions do not deviate significantly from a Gaussian shape. To help us find this value, we have fitted the density distributions of Figure 5 to a Gaussian expression, and have plotted the correlation coefficient of those fits as a function of $N_{G}$ in Figure 6. In this figure, it is easier to quantify the deviations from Gaussian shape both at low and at high resolutions. Applying the above criterion (points marked with arrows in Figure 6), we see that the distributions for $\mathrm{CCl}_{4}$ indeed start to deviate significantly from a Gaussian shape at the value of $N_{G} \approx 11$. For water, this point is observed at $N_{G} \approx 15$, which is lower than the value of 16 determined above - for $N_{G}=16$ the deviations are already quite pronounced. This hints at a possible limitation of the GIP method, as we will discuss in more detail below when we directly compare the results obtained with different methods. 


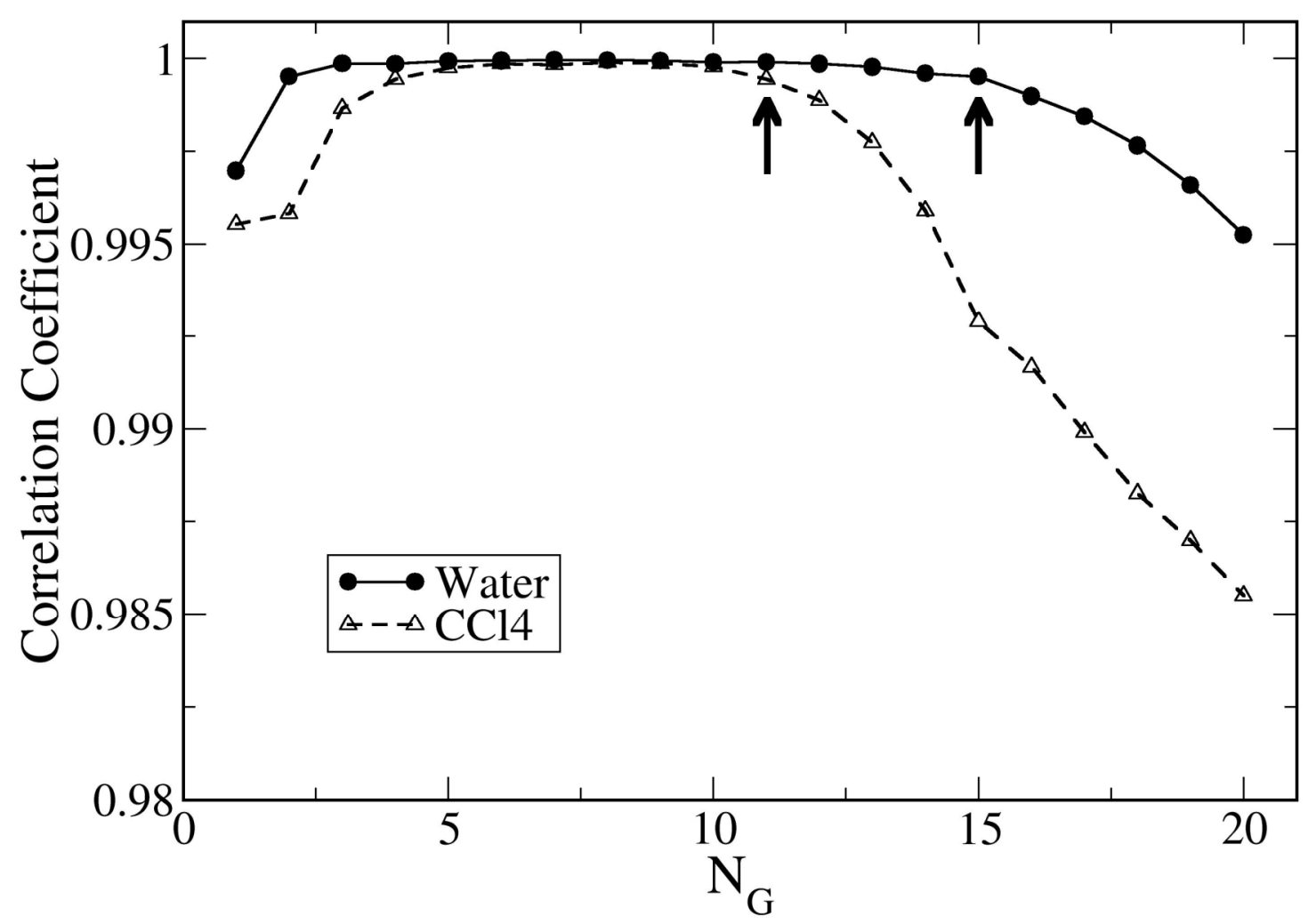

Figure 6 - Correlation coefficients obtained by fitting the interfacial density distributions obtained from the GIP method to a Gaussian expression, as a function of the grid resolution.

Another criterion one can use to find the optimal value of the grid resolution is to check the amount of overlap between the GIP distributions and the optimal ISM distributions (used here as a reference case). In other words, we compare the list of interfacial molecules computed using both methods for each configuration, and calculate the fraction of molecules that belong to both distributions simultaneously. The results of this procedure are shown in Figure 7 for the GIP distributions obtained using different values of $N_{G}$. As we can see, the maximum overlap with the ISM distribution is reached for the organic phase with $N_{G}=11$ and for water with $N_{G}=$ 16, which is in excellent agreement with the other criteria mentioned above. The percentage of overlap for $\mathrm{CCl}_{4}$ (about 90\%) is reasonable, but it is rather low for water (about 78\%). Once more, this is due to a limitation of the GIP method, as we will discuss below. 


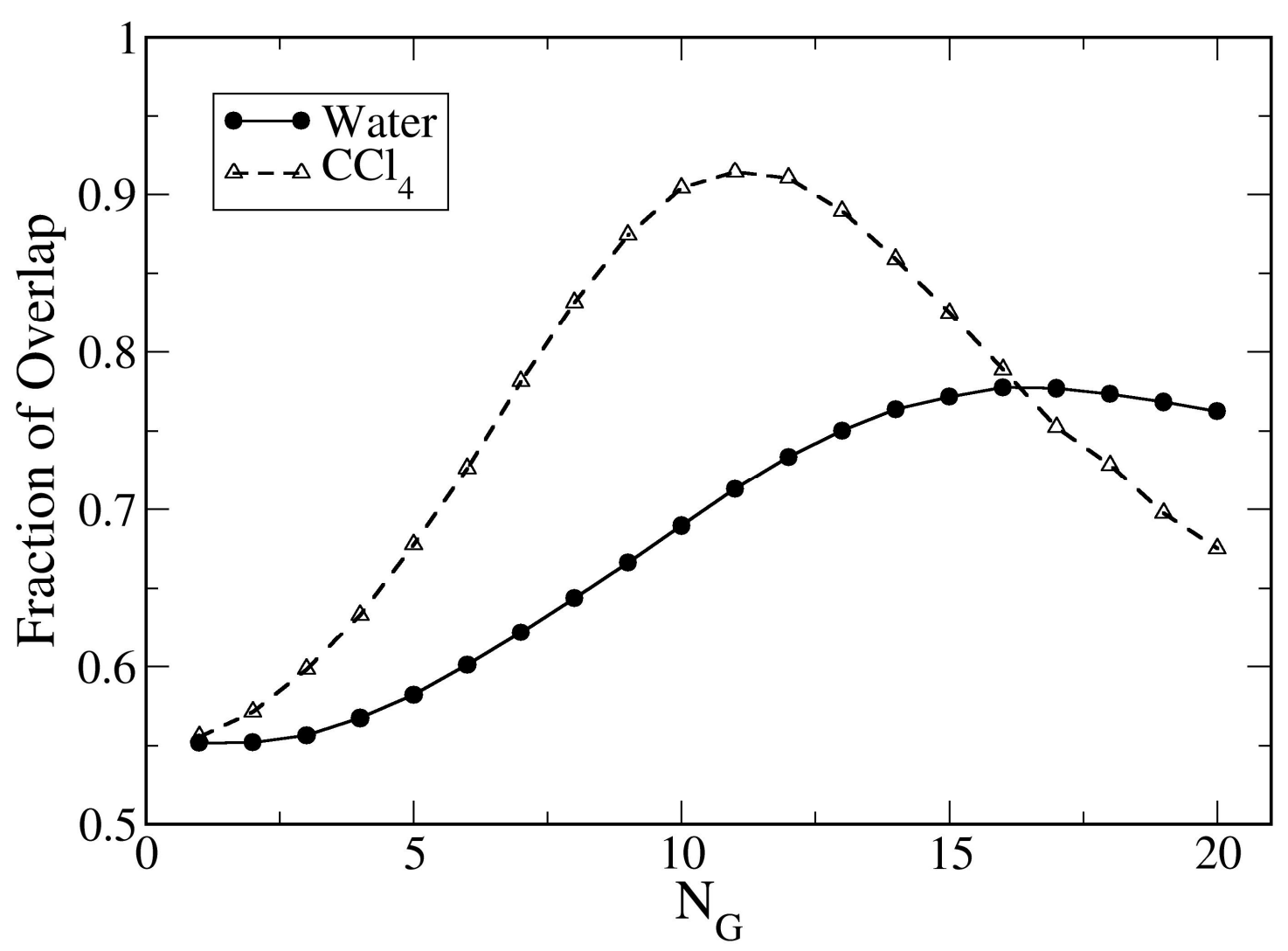

Figure 7 - Fraction of overlap between the optimal surface density distributions from the ISM method and the distributions obtained with the GIP method using different values of the grid resolution.

\subsection{The Identification of Truly Interfacial Molecules of Pártay et al.}

At the same time as the work of Jorge and Cordeiro was published, Pártay et al. [10] developed a method for identifying the set of interfacial molecules of the water/vapor interface. The so-called Identification of Truly Interfacial Molecules (ITIM) method, later extended to liquid/liquid interfaces [15], is based on constructing a grid of test lines that run perpendicularly to the interface. This is achieved by dividing the interfacial plane into a grid of $N_{t l} \times N_{t l}$ squares, much in the same way as in the GIP method. The main difference lies in the criterion for identifying the interfacial molecules - whereas in the GIP method the outermost site in each rectangular prism is selected, in the ITIM method interfacial sites are detected based on the intersection with a probe sphere of a predetermined radius $\left(R_{P}\right)$ whose center lies on each test line. In practice, for each test line a probe sphere is moved from the bulk of phase $i$ to the bulk 
of phase $j$ and is stopped as soon as it overlaps with an atomic site belonging to phase $j$. The site with which it overlapped is considered to belong to an interfacial molecule, and the procedure is then repeated for all the test lines.

As we will discuss in more detail in Section 3.5, the ITIM method has an important advantage over the GIP method - whereas in the latter decreasing the grid spacing below the characteristic site diameter is not physically reasonable, in the ITIM method the resolution of the grid can be increased indefinitely (within reason), provided the probe sphere radius retains a value of the order of the atomic size. However, this comes with the disadvantage of having two free parameters that can be adjusted $\left(N_{t l}\right.$ and $\left.R_{P}\right)$. It is thus important to examine how the results are affected by changes in those parameters. In Figure 8, we plot the dimensionless surface layer density obtained with a probe sphere radius of $0.2 \mathrm{~nm}$ (the value recommended by Pártay et al. [10] in their analysis) for increasing values of $N_{t l}$. Initially, $n_{S}$ increases proportionally to $N_{t l}{ }^{2}$ because the grid spacing is large and each test line detects a different interfacial molecule. However, the curves quickly level off to a plateau at high $N_{t l}$. In this regime, very few additional interfacial molecules are detected by introducing new test lines, which suggests the existence of a limiting value of $n_{S}$ for a given probe sphere radius. Although this limiting value can only strictly be reached with an infinite number of test lines, in practice a reasonable approximation can be obtained with a relatively small value of $N_{t l}$. To find the necessary number of test lines, we have fitted the data in Figure 8 to an expression of the type:

$$
n_{S}=\frac{A_{0} N_{t l}{ }^{2}}{A_{1}+{N_{t l}}^{2}},
$$

where $A_{0}$ and $A_{l}$ are fitting parameters. Equation (6) provides an excellent fit to the data, as can be seen in Figure 8. From the above expression and the values of the fitting parameters, one can estimate the number of test lines that are necessary to obtain a surface layer density that is within a given tolerance ( $t o l)$ of the limiting value. Using this criterion, the required value of $N_{t l}$ is given by: 


$$
N_{t l}=\sqrt{\frac{1-t o l}{t o l} A_{1}} .
$$

For a tolerance of $2 \%$ and a probe sphere radius of $0.2 \mathrm{~nm}$, we require 91 test lines for water and 48 for $\mathrm{CCl}_{4}$. For smaller probe spheres, the required number of test lines increased only slightly, and was never above 100 for water. Thus, we can confidently use $N_{t l}=100$ for water and $N_{t l}=$ 50 for $\mathrm{CCl}_{4}$. In the remainder of our analysis, however, we have adopted the conservative approach of using $N_{t l}=100$ for both components. It is worth noticing that this value is twice the value used by Pártay et al. $[10,15]$ - their value of $N_{t l}=50$ corresponds to a deviation of $\approx 6 \%$ from the limiting density for water.

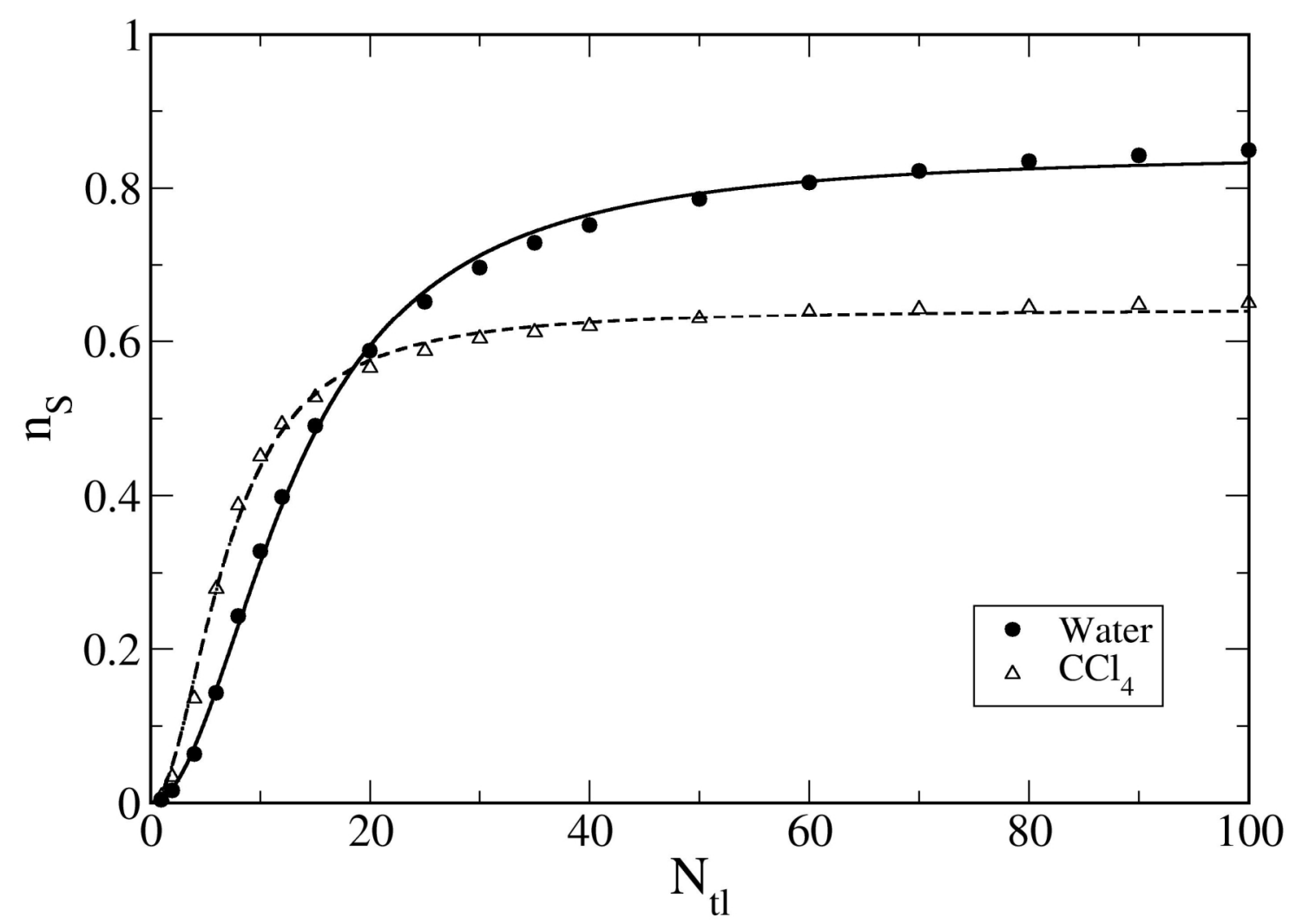

Figure 8 - Dimensionless surface layer density obtained with the ITIM method using a probe sphere radius of $0.2 \mathrm{~nm}$ and an increasing number of test lines. The lines are fits to the data using equation (6). 
Now that $N_{t l}$ has been fixed, we can analyze the effect of the probe sphere radius on the interfacial density distributions. These are shown in Figure 9 for several values of $R_{P}$. Similar to what was observed in the ISM and the GIP methods, the curves have an approximately Gaussian shape, and deviate from this shape at both very small and very large values of $R_{P}$. For large values of the probe sphere radius, only the outermost molecules are considered as interfacial, and the surface layer density is too low. When $R_{P}$ is too small, however, it can penetrate through small gaps in the interfacial layer and reach molecules that belong to the second molecular layer. When this happens, the distributions deviate from a Gaussian shape, and may even exhibit a shoulder in the direction of the bulk phase (see data for $\mathrm{CCl}_{4}$ in Figure 9). Analogously to the control parameters in the above methods $\left(n_{S}\right.$ in the ISM and $N_{G}$ in the GIP), an optimal value of $R_{P}$, providing the best possible description of the interfacial layer, is expected to exist. Intuitively, as discussed by Pártay et al. [10], this value should be of the same order as the characteristic site diameter in each phase.

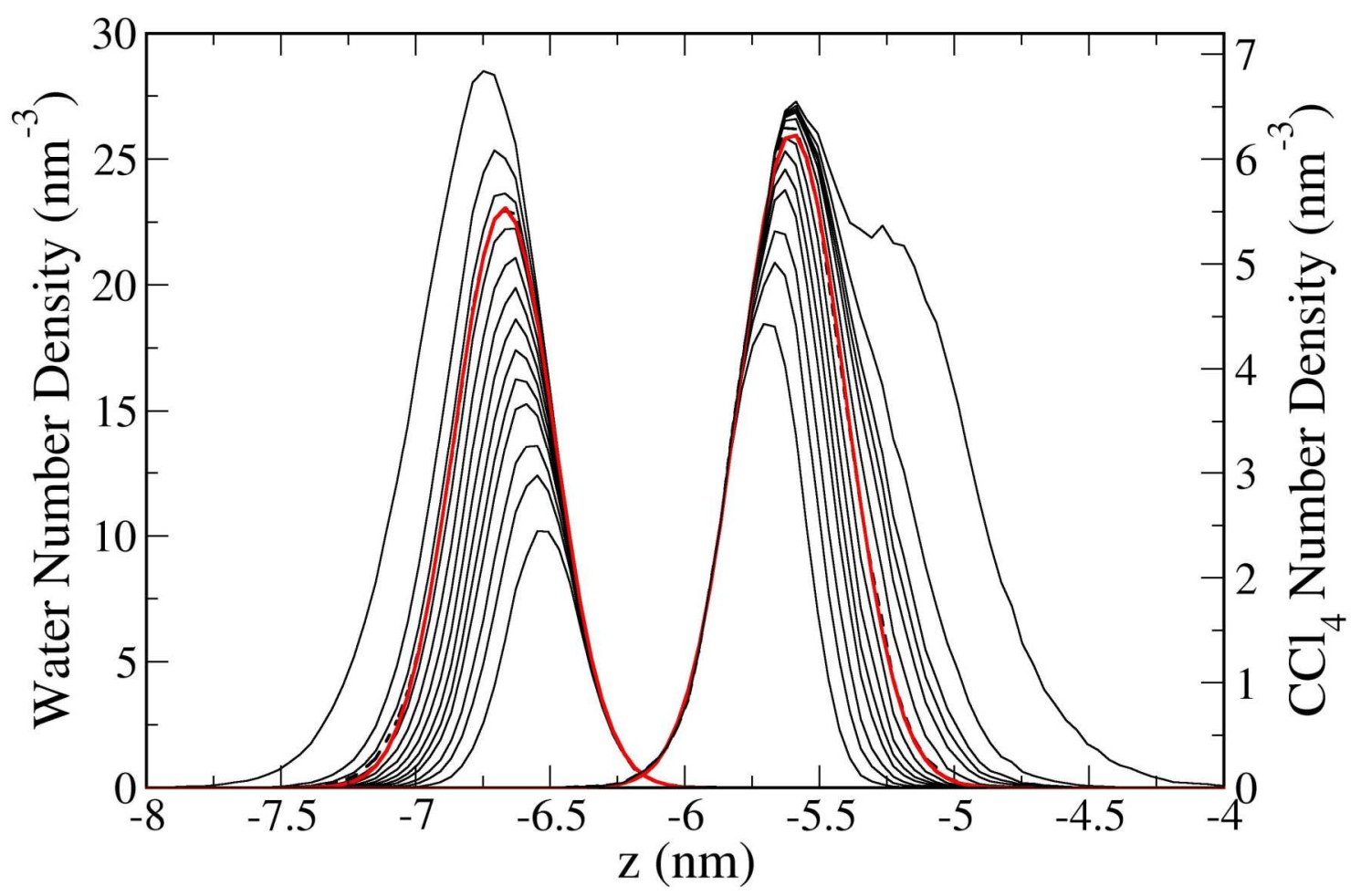


Figure 9 - Number density distributions of interfacial molecules for water (left curves and left axis) and $\mathrm{CCl}_{4}$ (right curves and right axis) obtained from the ITIM method with decreasing values of the probe sphere radius, from bottom to top: $R_{P}=0.5,0.4,0.35,0.3,0.275,0.25,0.225$, $0.2,0.175,0.15,0.137,0.125,0.1,0.05 \mathrm{~nm}$. The thick red lines are fits to a Gaussian distribution for selected curves (water $-0.137 \mathrm{~nm} ; \mathrm{CCl}_{4}-0.2 \mathrm{~nm}$ ), which are shown as dashed lines. The curves for $\mathrm{CCl}_{4}$ are shifted by $0.6 \mathrm{~nm}$ to the right for clarity.

As before, the deviations from a Gaussian shape can be more easily quantified by fitting the interfacial density distributions of Figure 9 to a Gaussian expression, and plotting the correlation coefficients as a function of the control parameter $\left(R_{P}\right.$ in this case). The results of this analysis are shown in Figure 10, where we can confirm the existence of an optimal range of probe sphere radii where the distributions have a truly Gaussian shape. Following the same reasoning as in section 2 , one can consider that the optimal probe sphere radius will be the smallest possible value of $R_{P}$ that still yields a Gaussian distribution (in other words, the radius that captures as many molecules belonging to the interfacial layer as possible, without capturing a significant number of molecules from the second layer). From Figure 10, we can see that this criterion is satisfied by $R_{P} \approx 0.125 \mathrm{~nm}$ for water and $R_{P} \approx 0.2 \mathrm{~nm}$ for $\mathrm{CCl}_{4}$ (points marked with arrows in Figure 10). 


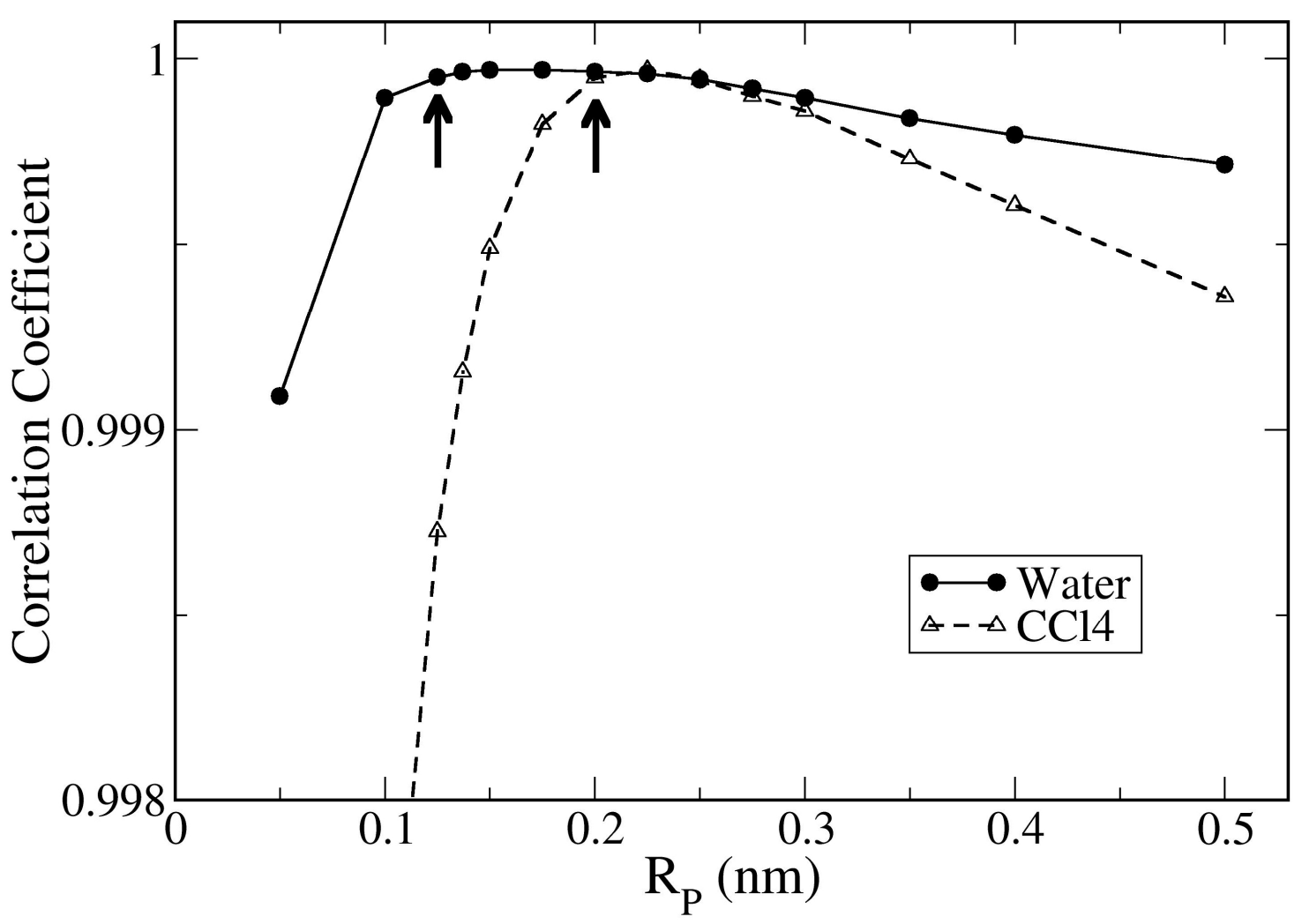

Figure 10 - Correlation coefficients obtained by fitting the interfacial density distributions obtained from the ITIM method to a Gaussian expression, as a function of the probe sphere radius.

Analogously to the above analysis of the GIP method, we can use the criterion of maximum overlap between the ITIM distributions and the reference ISM distribution to determine the optimal value of $R_{P}$. A plot of the overlap fractions is shown in Figure 11. Once again, there is a clear maximum in the overlap fraction for each phase, which is observed at the values of $R_{P} \approx 0.125 \mathrm{~nm}$ for water and $R_{P} \approx 0.2 \mathrm{~nm}$ for $\mathrm{CCl}_{4}$. These values are in excellent agreement with the above analysis based on the shape of the interfacial density distribution, which confirms the consistency of the ITIM method. It is also worth noticing that the maximum overlap percentages ( $88 \%$ for water and $95 \%$ for $\mathrm{CCl}_{4}$ ) are significantly higher than the corresponding values for the GIP method, which indicates that the ITIM is doing a better job than the latter at finding the molecules that actually belong to the surface layer. 


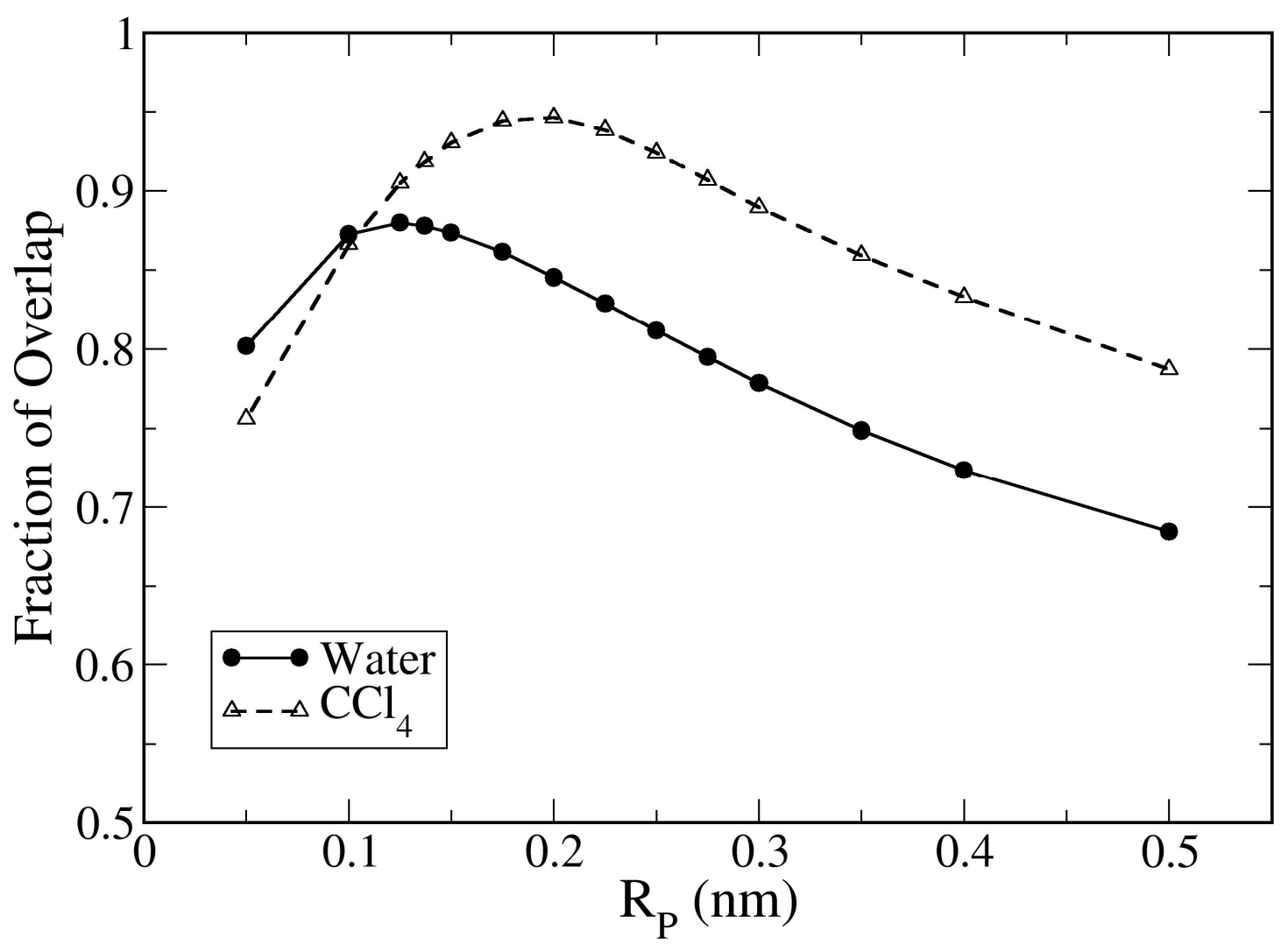

Figure 11 - Fraction of overlap between the optimal surface density distributions from the ISM method and the distributions obtained with the ITIM method using different values of the probe sphere radius.

It is worth noticing that the optimal probe sphere radius for water is somewhat below the value of $0.2 \mathrm{~nm}$ suggested by Pártay et al. [10]. Although in their later study of liquid/liquid interfaces [15] the authors suggested using the same probe sphere radius for both phases, our analysis clearly shows that a more accurate description of the system is obtained by using a different value of $R_{P}$ for each phase. This is physically reasonable, since the roughness of the interfacial layer is expected to depend on the molecular size and shape of the actual phase being analyzed. In our system, a smaller probe sphere is necessary to detect the smaller water molecules, while a larger probe sphere should be used for the larger $\mathrm{CCl}_{4}$ molecules. Interestingly, for both phases the optimal value of the probe diameter (twice the value of $R_{P}$ ) is about $80 \%$ of the respective characteristic diameter, and is close to the position of the first peak of the respective bulk radial distribution functions (RDFs) for water oxygens (peak located at 
$0.27 \mathrm{~nm}$ ) and for the $\mathrm{Cl}$ atoms of $\mathrm{CCl}_{4}$ (peak at $0.38 \mathrm{~nm}$ ). Although this agreement may be fortuitous, it nevertheless corroborates the physical meaning of the probe sphere radius in the ITIM method, and can perhaps be used as a guideline for finding the optimal $R_{P}$ without performing an exhaustive analysis, as done here. Finally, it is important to note that the values of the dimensionless surface density obtained with the ITIM method using the optimal probe sphere radii $\left(n_{S}=1.13\right.$ for water and $n_{S}=0.66$ for $\left.\mathrm{CCl}_{4}\right)$ are in excellent agreement with the results obtained using the self-consistent ISM in Section 3.1.

\subsection{The Surface Layer Identification of Chowdhary and Ladanyi}

The last method we consider in this paper is the Surface Layer Identification (SLI) technique, proposed by Chowdhary and Ladanyi [11] for the analysis of water/alkane interfaces. It is based on the notion that the surface layer of a given phase must include molecules of that liquid that are closest to the opposite phase. Thus, the core of the SLI method is a simple search over all pairs of atoms belonging to the two different phases - the site of phase $i$ that is closest to a given site of phase $j$ is considered as an interfacial site, and running over all sites of phase $j$ builds the surface layer of phase $i$. This procedure does indeed identify a set of the outermost molecules belonging to each phase, with the advantage, relative to the other methods, of not requiring the tuning of a control parameter. We will argue below, however, that such a control parameter is in fact necessary, due to the intrinsically fluid nature of the interface.

After searching for the sites with closest proximity to the opposite phase, the SLI method adds some more sites to the interfacial layer. For the organic phase, if a given molecule has a site that is considered to belong to the interface, all other sites on the same molecule that are closer to any water surface site were added to the list of organic interfacial sites. Here, instead, we consider all sites belonging to that molecule as interfacial (essentially, this is the same as considering the interfacial layer on a molecular basis, as was done above for the other methods). For the water phase, a slightly more complicated criterion is used: i) first, from all water molecules that are not yet part of the surface layer, those that are within a distance of 0.35 $\mathrm{nm}$ (we define this variable as the overlap distance, $D_{O}$ ) from a given surface site of the organic 
phase are chosen; ii) for each of these water molecules, a sphere with the corresponding molecular diameter is projected onto the $(x, y)$ plane; iii) if the projection of one of those water molecules overlaps with the corresponding projection of the organic site, then that water molecule is added to the interfacial layer. It should be noted here that the description of this part of the algorithm in the original paper [11] contains a typo - in the left column of page 15444, lines 7-10, where it reads "By projecting all water molecules $i$ within $3.5 \AA$ of each water surface molecule $j$ onto the $(x, z)$ and $(y, z)$ plane, if $i$ and $j$ overlap, molecule $i$ is added to the list of surface water molecules", it should read "By projecting all water molecules $i$ within $3.5 \AA$ of each organic surface molecule $j$ onto the $(x, y)$ plane, if $i$ and $j$ overlap, molecule $i$ is added to the list of surface water molecules". We have used here the correct implementation, as described above, following a personal communication by one of the authors [34].

The interfacial density distributions obtained using the SLI method are shown as thick dashed lines in Figure 12. For the organic component, the distribution is similar to the ones obtained using the previous methods, although the average surface density is somewhat lower $\left(n_{S}=0.59\right.$ for $\left.\mathrm{CCl}_{4}\right)$. For the water phase, however, only a very small number of molecules are included in the second step of the SLI method (compare distributions for $D_{O}=0$ and $D_{O}=0.35$ $n m$ in Figure 12), and the average surface density $\left(n_{S}=0.64\right.$ for water $)$ is much lower than with the other methods. These differences are due to the fact that the SLI method only considers as interfacial molecules those that are in direct contact with the opposite phase. In the case of water, this procedure ignores many molecules that are not in direct contact but that still belong to the interfacial layer. 


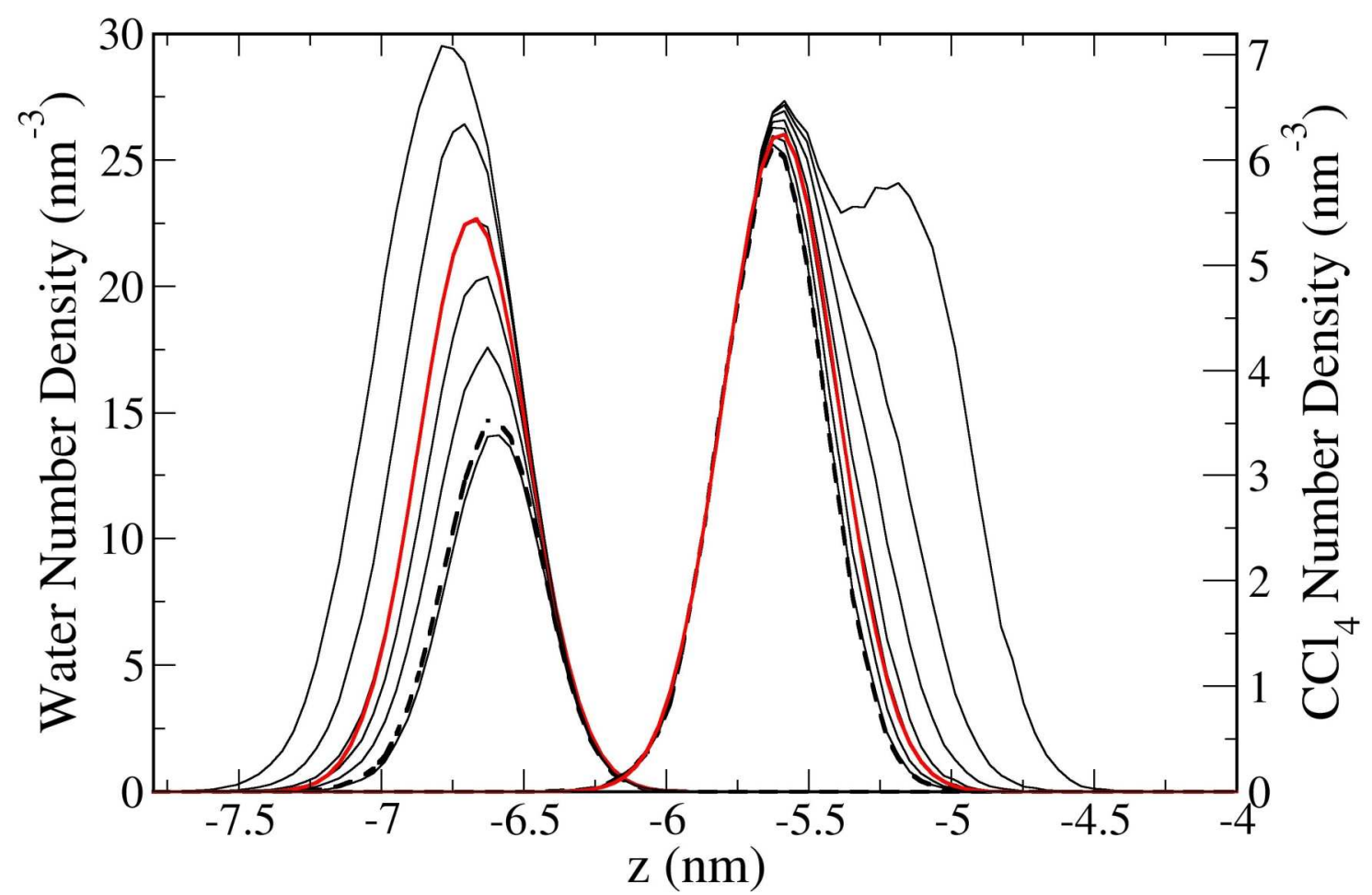

Figure 12 - Number density distributions of interfacial molecules for water (left curves and left axis) and $\mathrm{CCl}_{4}$ (right curves and right axis) obtained from the "extended" SLI method. The distributions are calculated for increasing values of the overlap distance, from bottom to top: $D_{O}$ $=0.0,0.35,0.4,0.45,0.5,0.6,0.7$ and (for $\mathrm{CCl}_{4}$ only) $0.9 \mathrm{~nm}$. The thick dashed lines correspond to the original version of the SLI method. The thick red lines are fits to a Gaussian distribution for selected curves (water $-0.5 \mathrm{~nm} ; \mathrm{CCl}_{4}-0.45 \mathrm{~nm}$ ). The curves for $\mathrm{CCl}_{4}$ are shifted by $0.6 \mathrm{~nm}$ to the right for clarity.

In the other three methods studied here, one is able to adjust a control parameter so that all molecules that belong to the interfacial layer are detected, at the same time minimizing the inclusion of molecules belonging to the second layer. In the ISM (Section 3.1), this is achieved by selecting the optimal surface layer density, $n_{S}$; for the GIP method (Section 3.2), the grid resolution can be tuned by manipulating $N_{G}$; for the ITIM procedure (Section 3.3), the control parameter is the probe sphere radius, $R_{P}$. This strongly suggests that a control parameter is in fact necessary to adequately cope with the fluid nature of liquid/liquid interfaces - without it, one cannot guarantee that all (and not more than) the interfacial molecules are detected. Thus, 
we have attempted to extend the SLI method by including a control parameter. The simplest choice is the overlap distance used for including additional water molecules in the second step of the original method. So, in our "extended" version of SLI (SLIx) the first step is identical to the original method, while the second step (applied now to both water and $\mathrm{CCl}_{4}$ ) adds molecules that are within a distance $D_{O}$ (where $D_{O}$ is now allowed to vary) from any surface site of the opposite phase determined in the first step, provided their projections onto the $(x, y)$ plane overlap.

The resulting distributions from the SLIx method are shown in Figure 12 for several values of $D_{O}$. As the overlap distance increases, more molecules are added to the interfacial list, and the surface density increases. Interestingly, we observed that this increase was practically linear for both components. In analogy with the other methods, one might expect the existence of an optimal value of $D_{O}$ that gives the best possible representation of the interfacial layer. To find out if this is the case, we have fitted the distributions of Figure 12 to a Gaussian expression, and have plotted the corresponding correlation coefficients in Figure 13. It can be seen that the distributions start off very close to a Gaussian shape (recall that here the first distribution, for $D_{O}=0 \mathrm{~nm}$, already corresponds to a relatively high surface density) but deviate from this shape for large values of the overlap distance. As before, we consider as an optimal choice the value of the control parameter right before a significant decrease is observed in the correlation coefficient. The corresponding values, marked with arrows in Figure 13, are $D_{O}=0.52 \mathrm{~nm}$ for water and $D_{O}=0.42 \mathrm{~nm}$ for $\mathrm{CCl}_{4}$, which correspond to average surface layer densities of $n_{S}=$ 1.14 for water and $n_{S}=0.64$ for $\mathrm{CCl}_{4}$. These results are now significantly closer to those obtained using the other methods. 


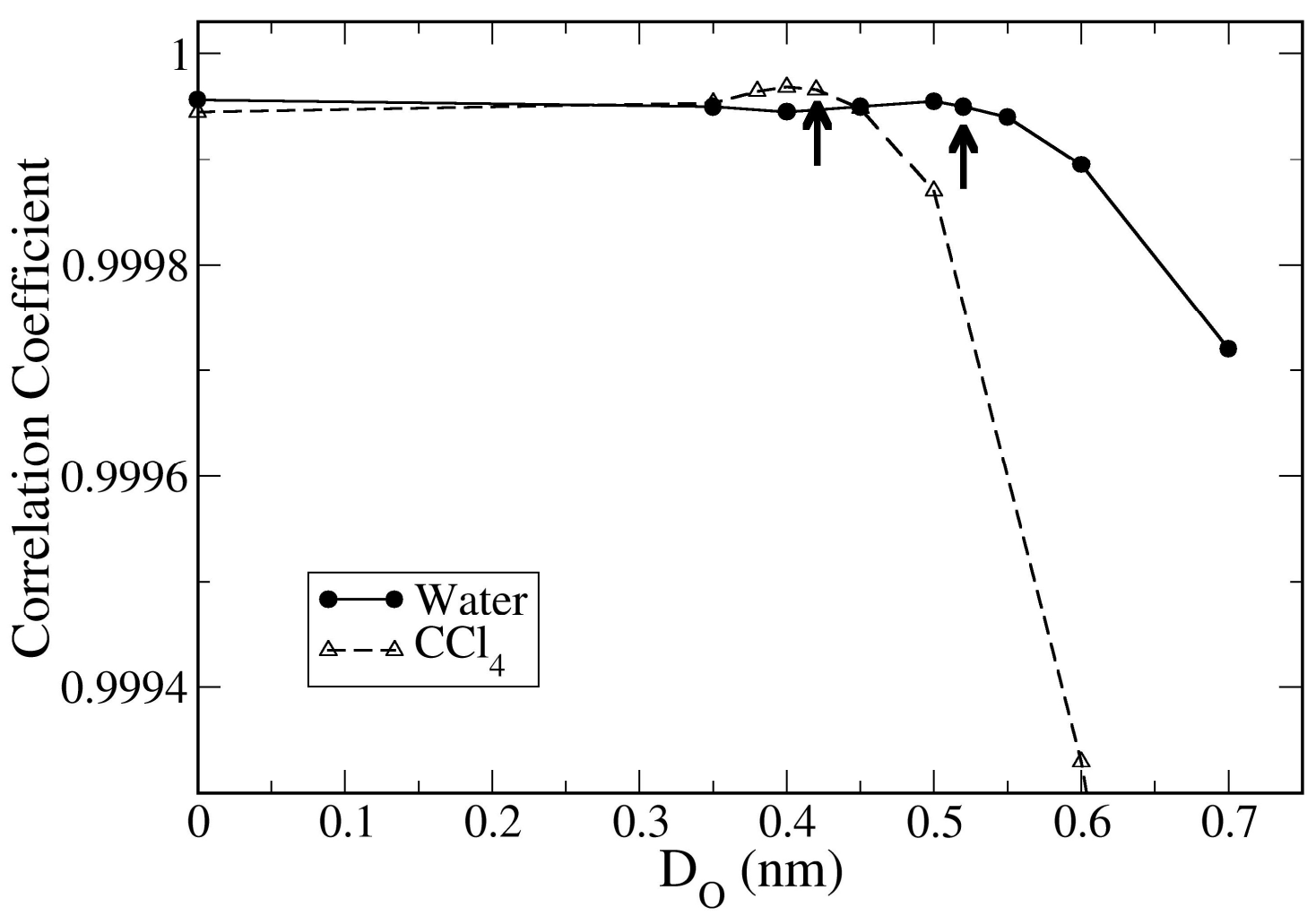

Figure 13 - Correlation coefficients obtained by fitting the interfacial density distributions obtained with the SLIx method to a Gaussian expression, as a function of the overlap distance.

The determination of the optimal value of the control parameter $D_{O}$ based on the shape of the interfacial density distribution, as shown in Figure 13, is less clear than for the other methods studied here. A clearer criterion is to choose the value of $D_{O}$ that maximizes the overlap with the reference ISM distribution. The degree of overlap for each phase, as a function of $D_{O}$ is plotted in Figure 14. As for the other methods, there exists a clear maximum in the overlap fraction. The optimal values thus obtained are $D_{O}=0.52 \mathrm{~nm}$ for water and $D_{O}=0.45$ $\mathrm{nm}$ for $\mathrm{CCl}_{4}$, which agree well with the values determined from the Gaussian fits. The maximum overlap percentages for the SLIx are identical to those obtained with the ITIM method (Figure 11). 


\subsection{Comparison between all methods.}

After finding the optimal values of the control parameters for each method, as well as optimizing their protocols, we are now in an ideal position to directly compare the results obtained from the four different approaches. In Figure 15 we plot the interfacial density distributions for both components calculated with the optimal values of control parameters for each method. In line with the discussion above, it is clear that the ISM, the ITIM and the SLIx methods all yield distributions that are in excellent agreement with each other. Quantitatively, the degree of overlap between these three methods is quite significant (95\% for $\mathrm{CCl}_{4}$ and almost $90 \%$ for water). The GIP method, however, gives results that are only slightly off for $\mathrm{CCl}_{4}$, but are in significant disagreement with the other methods for water (the agreement is not improved by using $N_{G}=15$ instead of 16 ). This is due to the limitation that the grid spacing in the GIP method cannot be increased beyond the order of atomic size. If this happens, the cross-sectional 
area of each prism is small enough to penetrate between gaps in the interfacial layer, and reach molecules that belong to the second layer, introducing a systematic error into the results. This effect is, of course, much more pronounced for water, due to its small size.

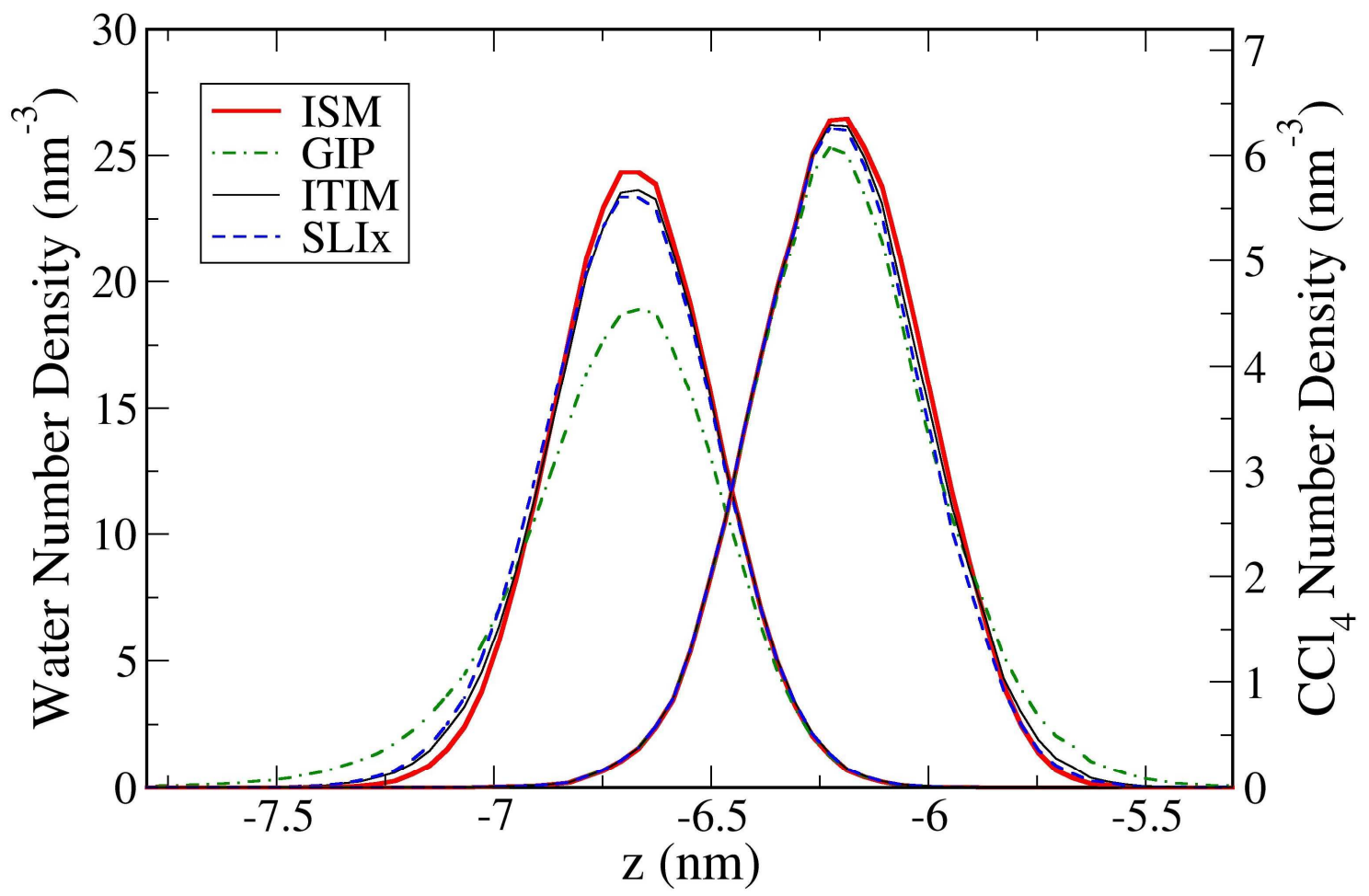

Figure 15 - Number density distributions of interfacial molecules for water (left curves and left axis) and $\mathrm{CCl}_{4}$ (right curves and right axis) obtained from the four methods studied here: ISM thick red line; GIP - dashed-dotted green line; ITIM - thin black line; SLIx - dashed blue line.

In the individual analysis of each method, we assessed the deviations of the interfacial density distributions from a Gaussian shape by plotting the correlation coefficients of the fits as a function of the control parameter of each method. However, it is also possible to plot all the data together by using the dimensionless surface layer density as a free variable (for the ISM method, $n_{S}$ itself is the control parameter). The results are shown in Figure 16 separately for the two components. As we can see, there is a good overlap between the ISM, the ITIM and the SLIx methods in the region of interest, and the optimal density corresponds, in all three cases, to the highest value before the curves start to deviate significantly from a Gaussian shape. For the 
GIP method, however, deviations from a Gaussian shape are already significant well before the optimal surface layer density is reached. Once again, the effect is much more pronounced in the case of the water surface.
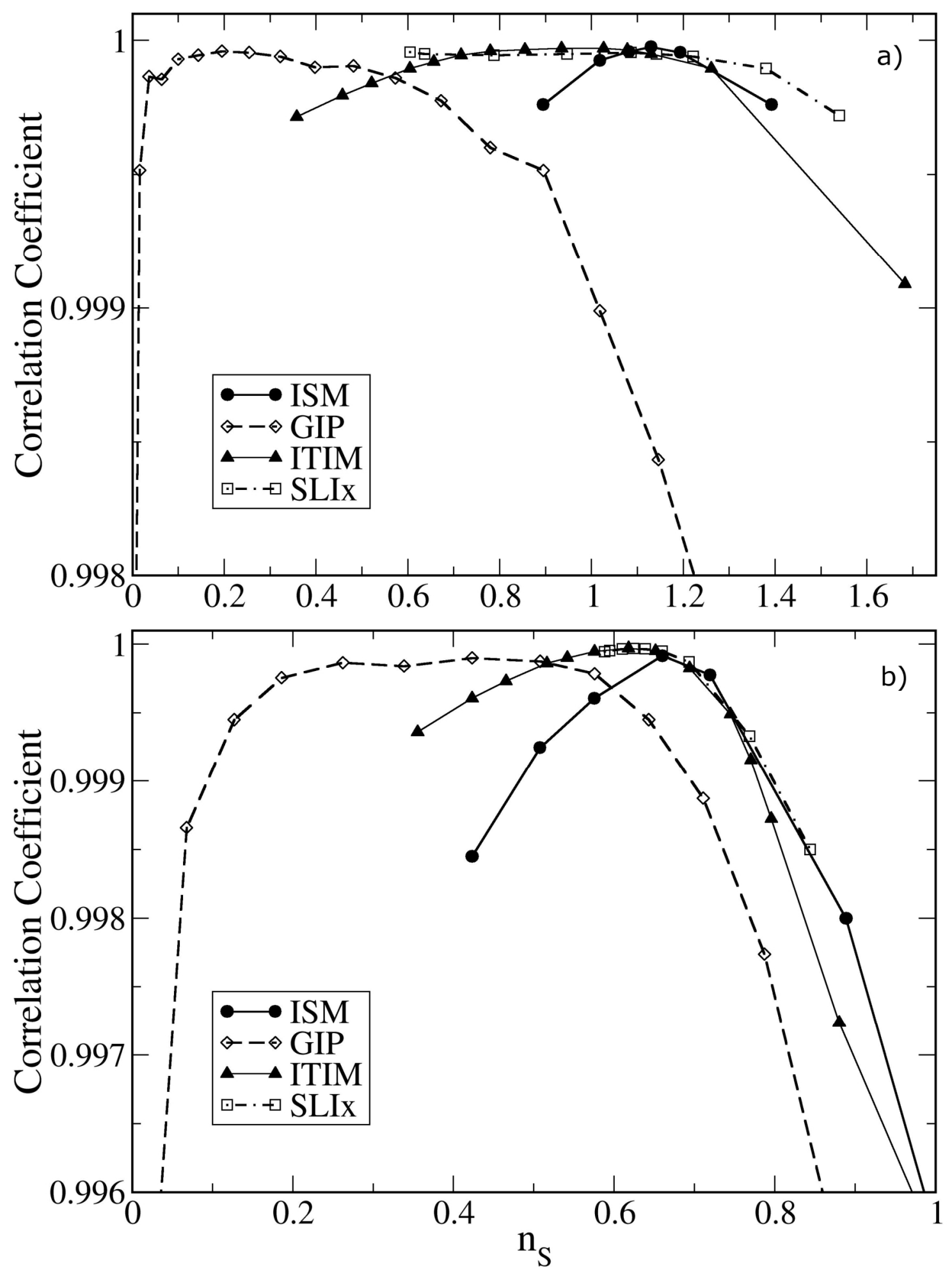
Figure 16 - Correlation coefficients obtained by fitting the interfacial density distributions obtained with the four methods studied here to a Gaussian expression, as a function of the dimensionless surface layer density, for: a) water; b) $\mathrm{CCl}_{4}$.

Finally, we can quantitatively assess the precision of the different methods in obtaining the correct surface layer densities for each phase, as well as their efficiency in terms of computational time (see Table 1). The densities obtained using the ISM method are taken as reference values, since this method was shown to be the most self-consistent procedure. Indeed, it permits direct and precise control of the surface layer density, and allows for a seemingly unambiguous way to determine the optimal value of the control parameter $n_{S}$, either using the techniques proposed in this paper (see Figures 2-4) or in the recent study by Chacón et al. [28]. Unfortunately, it is extremely computationally intensive (slower than the other methods by about two orders of magnitude) mostly because the set of equations (5) has to be solved at each step of the iteration. We have shown that it is possible to speed up the computation by using fewer wavevectors in the sum over Fourier components (although not much fewer than the value recommended by Chacón and Tarazona [21]) and starting from an initial estimate of the surface sites that is closer to the final result (within limits). However, even with this optimization, the method is still much more intensive than the others (the value shown in Table 1 corresponds to a run with $n_{M}=15$ for water, $n_{M}=10$ for $\mathrm{CCl}_{4}$, and $N_{0}=7$ ).

The GIP method yields surface layer densities that are somewhat below the ISM values, particularly for water, due to the limitations discussed above. Furthermore, it has the disadvantage of requiring an integer control parameter (e.g., it is impractical to divide the interfacial plane into a grid of, say, $15.3 \times 15.3$ squares). However, it is by far the fastest of all methods studied. Indeed, it was designed to yield a fast procedure to discretize the interface, so that intrinsic profiles could be easily calculated. The limitations of the method, however, introduce a systematic error when it is used to identify the true set of interfacial molecules. This happens because the GIP method searches for the outermost atomic centers located in each rectangular prism arising from the division of the interfacial plane into a grid. When the cross- 
section of the prism is smaller than the average spacing between two neighboring interfacial molecules (i.e., when the grid spacing goes below the characteristic atomic size), it becomes likely that a given prism does not "find" the center of an interfacial site, even if parts of the exclusion sphere of that site are indeed located within the prism. In this case, the search stops most often at the atomic center of a site belonging to the second layer beneath the interface. One possibility to circumvent this limitation is to introduce a criterion of overlap with the exclusion sphere of atomic sites in each prism, rather than searching only for the atomic centers. If the most efficient criterion, based on the overlap between two spheres, is used, the GIP method becomes conceptually identical to the ITIM.

The ITIM method gives results that are in remarkable agreement with the ISM, and is faster than the latter by a factor of $\approx 200$. This makes it the ideal choice for identifying the true set of interfacial molecules in a liquid/liquid system (and probably also in a liquid/vapor system). Finally, the SLI method yielded only a distribution of molecules that are in direct contact with the opposite phase, missing several molecules that do not obey this criterion but are nevertheless part of the interfacial layer. Modifying this method by introducing a control parameter (the overlap distance), we were able to obtain results in good agreement with the ISM values. However, the optimal value of $D_{O}$ is more difficult to identify than in the other methods, and we are unsure whether some well-defined physical meaning can be ascribed to it. Other choices of control parameter are obviously possible, but we did not pursue this avenue here. Furthermore, because interfacial molecules are chosen based on proximity to surface molecules of the opposite phase, a correlation between the two surfaces is introduced. Such a correlation is most likely unphysical, as has been demonstrated in a recent study by Hantal et al. [35]. The SLI method also has the important disadvantage of being inapplicable (in its present form) to liquid/vapor interfaces - it is not easy to determine which water molecules are in closest proximity to the vapor phase. In terms of efficiency, the SLIx method is about 1.5 times slower than the ITIM method, for a slightly lower precision. 
Table 1 - Comparison of the precision and computational efficiency of the different methods for finding the interfacial molecules.

\begin{tabular}{ccccc}
\hline Method & Control Parameter & $n_{S}$ Water & $n_{S} \mathrm{CCl}_{4}$ & Time $^{\mathrm{a}}(\mathrm{s})$ \\
\hline ISM & $n_{S}$ & 1.13 & 0.66 & 360.6 \\
GIP & $N_{G}$ & 1.02 & 0.64 & 0.046 \\
ITIM & $R_{P}$ & 1.13 & 0.65 & 1.751 \\
SLI & -- & 0.64 & 0.59 & 2.631 \\
SLIx & $D_{O}$ & 1.14 & 0.64 & 2.642 \\
& & &
\end{tabular}

\section{Conclusions}

In this paper we have presented a detailed comparison between several methods for intrinsic analysis of liquid interfaces, using as a prototype the water/ $\mathrm{CCl}_{4}$ interface, and focusing on their ability to consistently identify the set of molecules located at the surface of each phase. An important conclusion that arises from the application of these methods is the existence of a degree of molecular layering in the vicinity of the interface. Thus, a key aspect that controls the effectiveness of each method is a correct discrimination between molecules that belong to the first molecular layer (or interfacial layer) and those that are already part of the second molecular layer beneath the surface. We have shown that such discrimination requires the existence of a control parameter that can be fine-tuned to yield physically reasonable results. In the case of the ISM method, the control parameter is the surface layer density, and a recent study by Chacón et al. [28] demonstrates the self-consistency of different alternatives for determining the optimal value of this parameter. Here, we corroborate the conclusions of those authors, propose an additional alternative for finding the optimal surface density, and suggest possible ways to improve the computational efficiency of the method.

The ISM, however, suffers from the disadvantage of being highly computationally intensive, which emphasizes the need for more efficient methods that are able to accurately identify the true set of interfacial molecules. The ITIM method emerges from the present study 
as the most promising alternative to the ISM, since it is able to yield very similar results for the interfacial distributions but at a much lower computational cost. Nevertheless, we have shown here that care must be taken to ensure a sufficiently large density of test lines in the procedure, and to choose values of the probe sphere diameter (the ITIM control parameter) that are adequate for each phase. The optimal values of $R_{P}$ were seen to have a physical meaning, being about half of the position of the first peak of the characteristic bulk RDF for each fluid. The GIP method is even faster than the ITIM (by more than an order of magnitude), but suffers from a severe limitation: because it searches only for molecular centers in each position of the grid, the grid resolution cannot be increased beyond a characteristic atomic site, which introduces a systematic error in the identification of interfacial molecules. The ITIM method can be thought of as an extension of the GIP method using a criterion based on overlap between exclusion spheres, and is thus able to overcome the limitations of the latter. Finally, we have proposed an extension to the SLI method by introducing as a control parameter the overlap distance between atoms on opposite phases - the original SLI method possessed no control parameter and was thus able to identify only a subset of the interfacial molecules. This SLIx method does indeed yield results that are consistent with the ISM and the ITIM, but it is somewhat slower than the latter. More importantly, its application is currently restricted to liquid/liquid interfaces because it relies on a criterion that depends on proximity to molecules of the opposite phase, while the other three methods can be applied to liquid/vapor interfaces as well.

We should emphasize that the present paper only aims to compare the ability of the different methods for identifying the true set of interfacial molecules, and calculating the correct surface layer densities. In that sense, it should be expected that the methods that perform best (the ISM and the ITIM) were those that were designed with this objective in mind, and that is indeed the case. However, we say nothing about the ability of each method for computing intrinsic profiles. It is possible, for example, that the systematic error introduced by the GIP method in the detection of interfacial molecules becomes negligible when the profiles are computed. This issue assumes an even bigger importance if we consider that the main drawback of the ITIM method is that it currently proposes no procedure for the calculation of intrinsic 
profiles. We propose to analyze these aspects, related to the computation of intrinsic density profiles, in a subsequent publication.

Acknowledgements. The authors gratefully acknowledge Janamejaya Chowdhary for helpful insight regarding the SLI method. This project is partly supported by the Hungarian OTKA Foundation under project No. 75328, and by the Hungarian-Portuguese Intergovernmental Science and Technology Program. P. J. is a Bolyai János fellow of the Hungarian Academy of Sciences, which is gratefully acknowledged. M. J. and M. N. D. S. C. acknowledge financial support from Fundação para a Ciência e a Tecnologia - Portugal, through project PTDC/EQU-FTT/104195/2008.

\section{Supporting Information Available}

Optimization of the performance of the ISM, based on the tuning of several parameters. This information is available free of charge via the Internet at http://pubs.acs.org/.

\section{References}

[1] McFearin, C. L.; Beaman, D. K.; Moore, F. G.; Richmond, G. L. J. Phys. Chem. C 2009, $113,1171$.

[2] Benjamin, I. Annu. Rev. Phys. Chem. 1997, 48, 407.

[3] Schlossman, M. L. Curr. Opin. Colloid Interface Sci. 2002, 7, 235.

[4] Rowlinson, J; Widom, B. Molecular Theory of Capillarity; Clarendon Press: Oxford, 1982.

[5] Buff, F.; Lovett, R.; Stillinger, F. Phys. Rev. Lett. 1965, 15, 621.

[6] Mecke, K. R.; Dietrich, S. Phys. Rev. E 1999, 59, 6766.

[7] Stillinger, F. H. J. Chem. Phys. 1982, 76, 1087.

[8] Chacón, E.; Tarazona, P. Phys. Rev. Lett. 2003, 91, 166103.

[9] Jorge, M.; Cordeiro, M. N. D. S. J. Phys. Chem. C 2007, 111, 17612.

[10] Pártay, L. B.; Hantal, G.; Jedlovszky, P.; Vincze, A.; Horvai, G. J. Comput. Chem. 2008, $29,945$.

[11] Chowdhary, J.; Ladanyi, B. M. J. Phys. Chem. B 2006, 110, 15442. 
[12] Willard, A. P.; Chandler, D. J. Phys. Chem. B, 2010, 114, 1954.

[13] Jorgensen, W. L.; Chandrashekar, J.; Madura, J. D.; Impey, R.; Klein, M. L. J. Chem. Phys. 79, 926 (1983).

[14] McDonald, I. R.; Bounds, D. G.; Klein, M. L. Mol. Phys. 1982, 45, 521.

[15] Pártay, L. B.; Horvai, G.; Jedlovszky, P. Phys. Chem. Chem. Phys. 2008, 10, 4754.

[16] Lindahl, E.; Hess, B.; van der Spoel, D. J. Mol. Mod. 2001, 7, 306.

[17] Ryckaert, J. P.; Ciccotti, G.; Berendsen, H. J. C. J. Comput. Phys 1977,. 23, 327.

[18] Miyamoto, S.; Kollman, P. A. J. Comput. Chem. 1992, 13, 952.

[19] Berendsen, H. J. C.; Postma, J. P. M.; DiNola, A.; Haak, J. R. J. Chem. Phys. 1984, 81, 3684.

[20] Essman, U.; Perela, L.; Berkowitz, M. L.; Darden, T.; Lee, H.; Pedersen, L. G. J. Chem.

Phys. 1995, 103, 8577.

[21] Tarazona, P.; Chacón, E. Phys. Rev. B 2004, 70, 235407.

[22] Chacón, E.; Tarazona, P. J. Phys.: Condens. Matter 2005, 17, S3493.

[23] Chacón, E.; Tarazona, P.; Alejandre, J. J. Chem. Phys. 2006, 125, 014709.

[24] Bresme, F.; Chacón, E.; Tarazona, P. Phys. Chem. Chem. Phys. 2008, 10, 4704.

[25] Bresme, F.; Chacón, E.; Tarazona, P.; Tay, K. Phys. Rev. Lett. 2008, 101, 056102.

[26] Press, W.; Teukolsky, S.; Vetterling, W.; Flannery, B. Numerical recipes in C. 2 ed.; Cambridge University Press: Cambridge, 1992.

[27] Chowdhary, J.; Ladanyi, B. M. Phys. Rev. E 2008, 77, 031609.

[28] Chacón, E.; Fernández, E. M.; Duque, D.; Delgado-Buscalioni, R.; Tarazona, P. Phys. Rev. B 2009, 80, 195403.

[29] Linse, P. J. Chem. Phys. 1987, 86, 4177.

[30] Benjamin, I. J. Chem. Phys. 1992, 97, 1432.

[31] Weeks, J. D. J. Chem. Phys. 1977, 67, 3106.

[32] Fernandes, P. A.; Cordeiro, M. N. D. S.; Gomes, J. A. N. F. J. Phys. Chem. B 1999, 103, 8930.

[33] Jorge, M.; Cordeiro, M. N. D. S. J. Phys. Chem. B 2008, 112, 2415.

[34] Chowdhary, J.; personal communication. 
[35] Hantal, G.; Terleczky, P.; Horvai, G.; Nyulászi, L.; Jedlovszky, P. J. Phys. Chem. C 2009, $113,19263$. 


\section{TOC GRAPHIC}

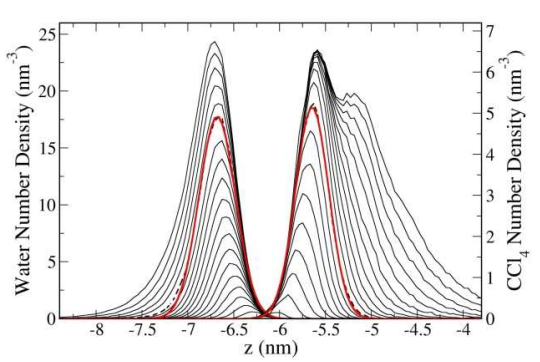

Number density distributions of interfacial molecules for water (left-hand side) and $\mathrm{CCl}_{4}$ (righthand side) obtained from the Grid-based Intrinsic Profile method with increasing grid resolution. There is an optimal resolution that yields the best possible description of the surface layer. 